\title{
Operators on Spaces of Bounded Vector-Valued Continuous Functions with Strict Topologies
}

\author{
Marian Nowak \\ Faculty of Mathematics, Computer Science and Econometrics, University of Zielona Góra, Ulica Szafrana 4A, \\ 65-516 Zielona Góra, Poland \\ Correspondence should be addressed to Marian Nowak; m.nowak@wmie.uz.zgora.pl
}

Received 30 June 2014; Accepted 26 August 2014

Academic Editor: Józef Banaś

Copyright (C) 2015 Marian Nowak. This is an open access article distributed under the Creative Commons Attribution License, which permits unrestricted use, distribution, and reproduction in any medium, provided the original work is properly cited.

Let $X$ be a completely regular Hausdorff space, and let $\left(E,\|\cdot\|_{E}\right)$ and $\left(F,\|\cdot\|_{F}\right)$ be Banach spaces. Let $C_{b}(X, E)$ be the space of all $E$-valued bounded, continuous functions defined on $X$, equipped with the strict topologies $\beta_{z}$, where $z=\sigma, \infty, p, \tau, t$. General integral representation theorems of $\left(\beta_{z},\|\cdot\|_{F}\right)$-continuous linear operators $T: C_{b}(X, E) \rightarrow F$ with respect to the corresponding operator-valued measures are established. Strongly bounded and $\left(\beta_{z},\|\cdot\|_{F}\right)$-continuous operators $T: C_{b}(X, E) \rightarrow F$ are studied. We extend to "the completely regular setting" some classical results concerning operators on the spaces $C(X, E)$ and $C_{o}(X, E)$, where $X$ is a compact or a locally compact space.

\section{Introduction and Terminology}

Throughout the paper let $\left(E,\|\cdot\|_{E}\right)$ and $\left(F,\|\cdot\|_{F}\right)$ be real Banach spaces, and let $E^{\prime}$ and $F^{\prime}$ denote the Banach duals of $E$ and $F$, respectively. By $B_{F^{\prime}}$ and $B_{E}$ we denote the closed unit ball in $F^{\prime}$ and $E$, respectively. By $\mathscr{L}(E, F)$ we denote the space of all bounded linear operators $U: E \rightarrow F$. Given a locally convex space $(L, \xi)$ by $(L, \xi)^{\prime}$ or $L_{\xi}^{\prime}$ we will denote its topological dual. We denote by $\sigma(L, K)$ the weak topology on $L$ with respect to a dual pair $\langle L, K\rangle$.

Assume that $X$ is a completely regular Hausdorff space. Let $C_{b}(X, E)$ stand for the Banach space of all bounded continuous, $E$-valued functions on $X$ provided with the uniform norm $\|\cdot\|$. We write $C_{b}(X)$ instead of $C_{b}(X, \mathbb{R})$. By $C_{b}(X, E)^{\prime}$ we denote the Banach dual of $C_{b}(X, E)$. For $f \in C_{b}(X, E)$ let $\tilde{f}(t)=\|f(t)\|_{E}$ for $t \in X$.

Let $\mathscr{B}$ (resp., $\mathscr{B} a$ ) be the algebra (resp., $\sigma$-algebra) of Baire sets in $X$, which is the algebra (resp., $\sigma$-algebra) generated by the class $\mathscr{Z}$ of all zero sets of functions of $C_{b}(X)$. By $\mathscr{P}$ we denote the family of all cozero sets in $X$. Let $B(\mathscr{B}, E)$ stand for the Banach space of all totally $\mathscr{B}$-measurable functions $f: X \rightarrow E$ (the uniform limits of sequences of $E$-valued $\mathscr{B}$-simple functions) provided with the uniform norm $\|\cdot\|$ (see $[1,2])$. We will write $B(\mathscr{B})$ instead of $B(\mathscr{B}, \mathbb{R})$.
Strict topologies $\beta_{z}$ on $C_{b}(X)$ and $C_{b}(X, E)$ (for $z=\sigma$, $\infty, p, \tau, t)$ play an important role in the topological measure theory (see [3-12] for definitions and more details). Recall that a subset $H$ of $C_{b}(X, E)$ is said to be solid if $f_{1} \in C_{b}(X, E)$ and $f_{2} \in H$ with $\tilde{f}_{1}(t) \leq \tilde{f}_{2}(t)$ for $t \in X$ imply that $f_{1} \in H$. Then $\beta_{z}$ are locally convex-solid topologies on $C_{b}(X, E)$; that is, they have a local base at 0 consisting of convex and solid sets (see [6, Theorem 8.1], [10, Theorem 5]). We have $\beta_{t} \subset$ $\beta_{\tau} \subset \beta_{\infty} \subset \beta_{\sigma} \subset \mathscr{T}_{\|\cdot\|}$ and $\beta_{t} \subset \beta_{p} \subset \beta_{\sigma}$. For a net $\left(f_{\alpha}\right)$ in $C_{b}(X, E), f_{\alpha} \rightarrow 0$ for $\beta_{z}$ if and only if $\tilde{f}_{\alpha} \rightarrow 0$ for $\beta_{z}$ in $C_{b}(X)($ see $[6,10])$.

Let $C_{b}(X) \otimes E$ stand for the algebraic tensor product of $C_{b}(X)$ and $E$; that is, $C_{b}(X) \otimes E$ is the space of all functions $\sum_{i=1}^{n}\left(u_{i} \otimes x_{i}\right)$, where $u_{i} \in C_{b}(X), x_{i} \in E$ for $i=1, \ldots, n$, and $\left(u_{i} \otimes x_{i}\right)(t)=u_{i}(t) x_{i}$ for $t \in X$. Then $C_{b}(X) \otimes E$ is dense in $\left(C_{b}(X, E), \beta_{z}\right)$ for $z=\infty, \tau, t$ (see $\left.[6,8]\right)$. Moreover, $C_{b}(X) \otimes$ $E$ is dense in $\left(C_{b}(X, E), \beta_{\sigma}\right)$ if $X$ or $E$ is a $D$-space (see [6, Theorem 5.2], [13]) and in $\left(C_{b}(X, E), \beta_{p}\right)$ if $X$ is real-compact (see [10, Theorem 7]).

Let $C_{r c}(X, E)$ denote the Banach space of all continuous functions $h: X \rightarrow E$ such that $h(X)$ is a relatively compact set in $E$, provided with the uniform norm $\|\cdot\|$. Then $C_{b}(X) \otimes$ $E \subset C_{r c}(X, E) \subset B(\mathscr{B}, E)$. 
Linear operators from the spaces $C_{r c}(X, E)$ and $C_{b}(X, E)$, equipped with the strict topologies $\beta_{z}(z=\sigma, \infty, \tau)$ to a locally convex space $(F, \xi)$, were studied by Katsaras and Liu [14], Aguayo-Garrido, Nova-Yanéz and Sanchez [15, 16], and Khurana [17]. In particular, Katsaras and Liu found an integral representation of weakly compact operators $S$ : $C_{r c}(X, E) \rightarrow F$ and characterizations of $\left(\beta_{z}, \xi\right)$-continuous and weakly compact operators $S: C_{r c}(X, E) \rightarrow F$ for $z=\sigma, \tau$ (see [14, Theorems 3, 4, 5]). Aguayo-Arrido and Nova-Yanéz derived a Riesz representation theorem for $\left(\beta_{z}, \xi\right)$-continuous and weakly compact operators $T: C_{b}(X, E) \rightarrow F$ for $z=$ $\infty, \tau$ in terms of their representing operator measures (see [15, Theorems 5 and 6]). If $X$ is a locally compact space, continuous operators on $C_{o}(X, E)$ were studied by Dobrakov (see [18]) and Mitter and Young (see [19]).

In this paper we develop the theory of continuous linear operators from $C_{b}(X, E)$, equipped with the strict topologies $\beta_{z}(z=\sigma, \infty, p, \tau, t)$ to a Banach space $\left(F,\|\cdot\|_{F}\right)$. In particular, we extend to "the completely regular setting" some classical results of Brooks and Lewis (see [20, Theorem 5], [21, Theorem 5.2], [22, Theorem 2.1]) concerning operators on the spaces $C(X, E)$ and $C_{o}(X, E)$, where $X$ is a compact or a locally compact space, respectively. In Section 2, using the device of embedding the space $B(\mathscr{B}, E)$ into $C_{r c}(X, E)^{\prime \prime}$ (the Banach bidual of $C_{r c}(X, E)$ ), we state the integral representation of bounded linear operators from $C_{r c}(X, E)$ to $F$. In Section 3 we derive general Riesz representation theorems for $\left(\beta_{z},\|\cdot\|_{F}\right)$-continuous linear operators $T: C_{b}(X, E) \rightarrow$ $F(z=\sigma, \infty, p, \tau, t)$ with respect to the corresponding measures $m: \mathscr{B} \rightarrow \mathscr{L}\left(E, F^{\prime \prime}\right)$ (see Theorems 9 and 14 below). Section 4 is devoted to the study of $\left(\beta_{\sigma},\|\cdot\|_{F}\right)$ continuous and strongly bounded operators $T: C_{b}(X, E) \rightarrow$ F.

\section{Integral Representation of Bounded Linear Operators on $C_{r c}(X, E)$}

Let $M(X)$ stand for the Banach lattice of all Baire measures on $\mathscr{B}$, provided with the norm $\|\nu\|=|\nu|(X)$ (= the total variation of $v$ ). Due to the Alexandrov representation theorem $C_{b}(X)^{\prime}$ can be identified with $M(X)$ through the lattice isomorphism $M(X) \ni \nu \mapsto \varphi_{\nu} \in C_{b}(X)^{\prime}$, where $\varphi_{\nu}(u)=\int_{X} u d v$ for $u \epsilon$ $C_{b}(X)$ and $\left\|\varphi_{\nu}\right\|=\|\nu\|$ (see [4, Theorem 5.1]).

By $M\left(X, E^{\prime}\right)$ we denote the set of all finitely additive measures $\mu: \mathscr{B} \rightarrow E^{\prime}$ with the following properties:

(i) for each $x \in E$, the function $\mu_{x}: \mathscr{B} \rightarrow \mathbb{R}$ defined by $\mu_{x}(A)=\mu(A)(x)$ belongs to $M(X)$,

(ii) $|\mu|(X)<\infty$, where $|\mu|(A)$ stands for the variation of $\mu$ on $A \in \mathscr{B}$.

In view of [23, Theorem 2.5] $C_{r c}(X, E)^{\prime}$ can be identified with $M\left(X, E^{\prime}\right)$ through the linear mapping $M\left(X, E^{\prime}\right) \ni \mu \mapsto$ $\Phi_{\mu} \in C_{r c}(X, E)^{\prime}$, where $\Phi_{\mu}(h)=\int_{X} h d \mu$ for $h \in C_{r c}(X, E)$ and $\left\|\Phi_{\mu}\right\|=|\mu|(X)$. Then one can embed $B(\mathscr{B}, E)$ into $C_{r c}(X, E)^{\prime \prime}$ by the mapping $\pi: B(\mathscr{B}, E) \rightarrow C_{r c}(X, E)^{\prime \prime}$, where for $g \epsilon$ $B(\mathscr{B}, E)$,

$$
\pi(g)\left(\Phi_{\mu}\right):=\int_{X} g d \mu \quad \text { for } \mu \in M\left(X, E^{\prime}\right) .
$$

Let $i_{F}: F \rightarrow F^{\prime \prime}$ denote the canonical embedding; that is, $i_{F}(y)\left(y^{\prime}\right)=y^{\prime}(y)$ for $y \in F, y^{\prime} \in F^{\prime}$. Moreover, let $j_{F}$ : $i_{F}(F) \rightarrow F$ stand for the left inverse of $i_{F}$; that is, $j_{F} \circ i_{F}=i d_{F}$.

Assume that $S: C_{r c}(X, E) \rightarrow F$ is a bounded linear operator. Let

$$
\widehat{S}:=S^{\prime \prime} \circ \pi: B(\mathscr{B}, E) \longrightarrow F^{\prime \prime},
$$

where $S^{\prime}: F^{\prime} \rightarrow C_{r c}(X, E)^{\prime}$ and $S^{\prime \prime}: C_{r c}(X, E)^{\prime \prime} \rightarrow F^{\prime \prime}$ denote the conjugate and biconjugate operators of $S$, respectively. Then we can define a measure $m: \mathscr{B} \rightarrow \mathscr{L}\left(E, F^{\prime \prime}\right)$ (called a representing measure of $S$ ) by

$$
\begin{array}{r}
m(A)(x):=\widehat{S}\left(\mathbb{1}_{A} \otimes x\right)=\left(S^{\prime \prime} \circ \otimes \pi\right)\left(\mathbb{1}_{A} \otimes x\right) \\
\text { for } A \in \mathscr{B}, x \in E .
\end{array}
$$

Then $\widetilde{m}(X)<\infty$, where the semivariation $\widetilde{m}(A)$ of $m$ on $A \in \mathscr{B}$ is defined by $\widetilde{m}(A):=\sup \left\|\sum m\left(A_{i}\right)\left(x_{i}\right)\right\|_{F^{\prime \prime}}$, where the supremum is taken over all finite $\mathscr{B}$-partitions $\left(A_{i}\right)$ of $A$ and $x_{i} \in B_{E}$ for each $i$. For $y^{\prime} \in F^{\prime}$ let us put

$$
m_{y^{\prime}}(A)(x):=(m(A)(x))\left(y^{\prime}\right) \quad \text { for } A \in \mathscr{B}, x \in E .
$$

Let $\left|m_{y^{\prime}}\right|(A)$ stand for the variation of $m_{y^{\prime}}$ on $A$. Then (see [1, Section 4, Proposition 5])

$$
\widetilde{m}(A)=\sup \left\{\left|m_{y^{\prime}}\right|(A): y^{\prime} \in B_{F^{\prime}}\right\} .
$$

The following general properties of the operator $\widehat{S}$ : $B(\mathscr{B}, E) \rightarrow F^{\prime \prime}$ are well known (see [1, Section 6], [2, Section $1],[13,24])$ :

$$
\widehat{S}(g)=\int_{X} g d m \text { for } g \in B(\mathscr{B}, E),\|\widehat{S}\|=\widetilde{m}(X),
$$

and for each $y^{\prime} \in F^{\prime}$,

$$
\widehat{S}(g)\left(y^{\prime}\right)=\int_{X} g d m_{y^{\prime}} \quad \text { for } g \in B(\mathscr{B}, E) .
$$

For $A \in \mathscr{B}$ let

$$
\int_{A} g d m:=\int_{X} \mathbb{1}_{A} g d m \text { for } g \in B(\mathscr{B}, E) .
$$

From the general properties of $\widehat{S}$ it follows that

$$
\begin{gathered}
\widehat{S}\left(C_{r c}(X, E)\right) \subset i_{F}(F), \\
S(h)=j_{F}\left(\int_{X} h d m\right) \quad \text { for } h \in C_{r c}(X, E) .
\end{gathered}
$$

Hence for each $y^{\prime} \in F^{\prime}$ we get

$$
y^{\prime}(S(h))=\int_{X} h d m_{y^{\prime}} \quad \text { for } h \in C_{r c}(X, E),
$$


and hence $m_{y^{\prime}} \in M\left(X, E^{\prime}\right)$. Moreover, we have

$$
\begin{aligned}
\|S\| & =\left\|S^{\prime}\right\| \\
& =\sup \left\{\left\|S^{\prime}\left(y^{\prime}\right)\right\|: y^{\prime} \in B_{F^{\prime}}\right\} \\
& =\sup \left\{\left\|y^{\prime} \circ S\right\|: y^{\prime} \in B_{F^{\prime}}\right\} \\
& =\sup \left\{\left\|\Phi_{m_{y^{\prime}}}\right\|: y^{\prime} \in B_{F^{\prime}}\right\} \\
& =\sup \left\{\left|m_{y^{\prime}}\right|(X): y^{\prime} \in B_{F^{\prime}}\right\},
\end{aligned}
$$

and using (5) we get

$$
\|S\|=\widetilde{m}(X) .
$$

By $M\left(X, \mathscr{L}\left(E, F^{\prime \prime}\right)\right)$ we will denote the space of all measures $m: \mathscr{B} \rightarrow \mathscr{L}\left(E, F^{\prime \prime}\right)$ such that $\widetilde{m}(X)<\infty$ and $m_{y^{\prime}} \in M\left(X, E^{\prime}\right)$ for each $y^{\prime} \in F^{\prime}$. Thus the representing measure $m$ of $S$ belongs to $M\left(X, \mathscr{L}\left(E, F^{\prime \prime}\right)\right)$.

For any $x \in E$ define

$$
\begin{gathered}
S_{x}(u):=S(u \otimes x) \quad \text { for } u \in C_{b}(X), \\
m_{x}(A):=m(A)(x) \quad \text { for } A \in \mathscr{B} .
\end{gathered}
$$

Then $S_{x}: C_{b}(X) \rightarrow F$ is a bounded linear operator. Let $\chi:$ $B(\mathscr{B}) \rightarrow C_{b}(X)^{\prime \prime}$ stand for the canonical embedding; that is, for $u \in B(\mathscr{B})$,

$$
\chi(u)\left(\varphi_{\nu}\right)=\int_{X} u d v \quad \text { for } v \in M(X)
$$

Let

$$
\widehat{S}_{x}:=\left(S_{x}\right)^{\prime \prime} \circ \chi: B(\mathscr{B}) \longrightarrow F^{\prime \prime} .
$$

Then

$$
\begin{gathered}
\widehat{S}_{x}\left(C_{b}(X)\right) \subset i_{F}(F), \\
S_{x}(u)=j_{F}\left(\widehat{S}_{x}(u)\right) \quad \text { for } u \in C_{b}(X) .
\end{gathered}
$$

The following lemma will be useful.

Lemma 1. Let $S: C_{r c}(X, E) \rightarrow F$ be a bounded linear operator. Then $S^{\prime \prime}\left(\pi\left(\mathbb{1}_{A} \otimes x\right)\right)=\left(S_{x}\right)^{\prime \prime}\left(\chi\left(\mathbb{1}_{A}\right)\right)$ for any $x \in E$ and $A \in \mathscr{B}$.

Proof. Let $y^{\prime} \in F^{\prime}$. Then for each $u \in C_{b}(X)$,

$$
\begin{aligned}
\left(y^{\prime} \circ S_{x}\right)(u) & =y^{\prime}(S(u \otimes x)) \\
& =\int_{X}(u \otimes x) d m_{y^{\prime}}=\int_{X} u d m_{x, y^{\prime}} \\
& =\varphi_{m_{x, y^{\prime}}}(u) .
\end{aligned}
$$

Hence we have

$$
\begin{aligned}
& \left(S_{x}\right)^{\prime \prime}\left(\chi\left(\mathbb{1}_{A}\right)\right)\left(y^{\prime}\right) \\
& \quad=\chi\left(\mathbb{1}_{A}\right)\left(S_{x}^{\prime}\left(y^{\prime}\right)\right) \\
& \quad=\chi\left(\mathbb{1}_{A}\right)\left(y^{\prime} \circ S_{x}\right)=\chi\left(\mathbb{1}_{A}\right)\left(\varphi_{m_{x, y^{\prime}}}\right) \\
& \quad=\int_{X} \mathbb{1}_{A} d m_{x, y^{\prime}}=m_{x, y^{\prime}}\left(\mathbb{1}_{A}\right)=m_{x}\left(\mathbb{1}_{A}\right)\left(y^{\prime}\right) .
\end{aligned}
$$

On the other hand, for each $h \in C_{r c}(X, E),\left(y^{\prime} \circ S\right)(h)=$ $\int_{X} h d m_{y^{\prime}}=\Phi_{m_{y^{\prime}}}(h)$, and hence

$$
\begin{aligned}
S^{\prime \prime} & \left(\pi\left(\mathbb{1}_{A} \otimes x\right)\right) \\
& =\left(\mathbb{1}_{A} \otimes x\right)\left(S^{\prime}\left(y^{\prime}\right)\right)=\pi\left(\mathbb{1}_{A} \otimes x\right)\left(y^{\prime} \circ S\right) \\
& =\pi\left(\mathbb{1}_{A} \otimes x\right)\left(\Phi_{m_{y^{\prime}}}\right)=\Phi_{m_{y^{\prime}}}\left(\mathbb{1}_{A} \otimes x\right) \\
& =\int_{X}\left(\mathbb{1}_{A} \otimes x\right) d m_{y^{\prime}}=m_{y^{\prime}}(A)(x)=m_{x}\left(\mathbb{1}_{A}\right)\left(y^{\prime}\right) .
\end{aligned}
$$

It follows that $S^{\prime \prime}\left(\pi\left(\mathbb{1}_{A} \otimes x\right)\right)=\left(S_{x}\right)^{\prime \prime}\left(\chi\left(\mathbb{1}_{A}\right)\right)$, as desired.

From Lemma 1 for $A \in \mathscr{B}$ and $x \in E$ we get

$$
m_{x}(A):=\widehat{S}\left(\mathbb{1}_{A} \otimes x\right)=S^{\prime \prime}\left(\pi\left(\mathbb{1}_{A} \otimes x\right)\right)=\left(S_{x}\right)^{\prime \prime}\left(\chi\left(\mathbb{1}_{A}\right)\right) ;
$$

that is,

$$
m_{x}(A)=\widehat{S}_{x}\left(\mathbb{1}_{A}\right), \quad \widehat{S}_{x}(u)=\int_{X} u d m_{x} \quad \text { for } u \in B(\mathscr{B}) .
$$

Now we are ready to prove the following Bartle-DunfordSchwartz type theorem (see [25, Theorem 5, pages 153-154]).

Theorem 2. Let $S: C_{r c}(X, E) \rightarrow F$ be a bounded linear operator and let $M\left(X, \mathscr{L}\left(E, F^{\prime \prime}\right)\right)$ be its representing measure. Then for each $x \in E$ the following statements are equivalent.

(i) $S_{x}: C_{b}(X) \rightarrow F$ is weakly compact.

(ii) $m(A)(x) \in i_{F}(F)$ for each $A \in \mathscr{B}$ and $\left\{j_{F}(m(A)(x))\right.$ : $A \in \mathscr{B}\}$ is a relatively weakly compact set in $F$.

(iii) $m_{x}: \mathscr{B} \rightarrow F^{\prime \prime}$ is strongly bounded.

Proof. (i) $\Rightarrow$ (ii) Assume that $S_{x}$ is weakly compact. Then by the Gantmacher theorem $\left(S_{x}\right)^{\prime \prime}\left(C_{b}(X)^{\prime \prime}\right) \subset i_{F}(F)$ and $\left(S_{x}\right)^{\prime \prime}$ : $C_{b}(X)^{\prime \prime} \rightarrow F^{\prime \prime}$ is weakly compact (see [26, Theorem 17.2]). Hence $\widehat{S}_{x}(B(\mathscr{B})) \subset i_{F}(F)$ and $\widehat{S}_{x}: B(\mathscr{B}) \rightarrow F^{\prime \prime}$ is weakly compact. In view of (21) for each $x \in E, m_{x}(A) \in i_{F}(F)$ for $A \in \mathscr{B}$ and $m_{x}: \mathscr{B} \rightarrow F^{\prime \prime}$ is strongly bounded (see [25, Theorem 1, page 148]). It follows that $\left\{j_{F}(m(A)(x)): A \in \mathscr{B}\right\}$ is a relatively weakly compact subset of $F$ (see [24, Theorem 7]).

(ii) $\Rightarrow$ (iii) It follows from [24, Theorem 7].

(iii) $\Rightarrow$ (i) Assume that $m_{x}: \mathscr{B} \rightarrow F^{\prime \prime}$ is strongly bounded. Then by $(21) \widehat{S}_{x}: B(\mathscr{B}) \rightarrow F^{\prime \prime}$ is weakly compact and in view of (16) we derive that $S_{x}$ is weakly compact. 


\section{Integral Representation of Continuous Linear Operators on $C_{b}(X, E)$}

The spaces of all $\sigma$-additive, $u$-additive, perfect, $\tau$-additive, and tight members of $M(X)$ will be denoted by $M_{\sigma}(X)$, $M_{\infty}(X), M_{p}(X), M_{\tau}(X)$, and $M_{t}(X)$, respectively (see $\left.[3,4]\right)$. Then $\left(C_{b}(X), \beta_{z}\right)^{\prime}=\left\{\varphi_{v}: v \in M_{z}(X)\right\}$ for $z=\sigma, \infty, p, \tau, t$.

For the integration theory of functions $f \in C_{b}(X, E)$ with respect to $\mu \in M_{z}\left(X, E^{\prime}\right)$ we refer the reader to [6, page 197], [5, Definition 3.10], [27, page 375]. For $z=\sigma, \infty, p, \tau$, $t$ let

$$
\begin{aligned}
& M_{z}\left(X, E^{\prime}\right) \\
& \quad:=\left\{\mu \in M\left(X, E^{\prime}\right): \mu_{x} \in M_{z}(X) \text { for each } x \in E\right\} .
\end{aligned}
$$

Then $|\mu| \in M_{z}(X)$ if $\mu \in M_{z}\left(X, E^{\prime}\right)$ (see [5, Proposition 3.9], [6, Theorem 3.1], [10, Theorem 1]). For $\Phi \in C_{b}(X, E)^{\prime}$ let us put, for $u \in C_{b}(X)^{+}$,

$$
|\Phi|(u):=\sup \left\{|\Phi(f)|: f \in C_{b}(X, E), \tilde{f} \leq u\right\} .
$$

It is known that $|\Phi|: C_{b}(X)^{+} \rightarrow \mathbb{R}^{+}$is additive and positively homogeneous and can be extended to a linear functional on $C_{b}(X)$ (denoted by $|\Phi|$ again) by $|\Phi|(u)=|\Phi|\left(u^{+}\right)-|\Phi|\left(u^{-}\right)$ for $u \in C_{b}(X)$.

Theorem 3. Assume that $z=\sigma$ and $C_{b}(X) \otimes E$ is dense in $\left(C_{b}(X, E), \beta_{\sigma}\right)$ (resp., $z=\infty ; z=p$ and $C_{b}(X) \otimes E$ is dense in $\left.\left(C_{b}(X, E), \beta_{p}\right) ; z=\tau ; z=t\right)$. Then the following statements hold.

(i) For a linear functional $\Phi$ on $C_{b}(X, E)$ the following conditions are equivalent.

(a) $\Phi$ is $\beta_{z}$-continuous.

(b) There exists a unique $\mu \in M_{z}\left(X, E^{\prime}\right)$ such that

$$
\Phi(f)=\Phi_{\mu}(f)=\int_{X} f d \mu \quad \text { for } f \in C_{b}(X, E) .
$$

(ii) For $\mu \in M_{z}\left(X, E^{\prime}\right),\left|\Phi_{\mu}\right|(u)=\int_{X} u d|\mu|=\varphi_{|\mu|}(u)$ for $u \in C_{b}(X)$.

Proof. (i) See [6, Theorems 5.3 and 4.2, Corollary 3.9], [5, Theorem 3.13], and [10, Theorem 8].

(ii) See [6, Theorem 2.1].

Assume that $\mathscr{M}$ is a subset of $M_{z}\left(X, E^{\prime}\right)$ and $\sup _{\mu \in \mathscr{M}}|\mu|(X)<\infty$, where $z=\sigma, \infty, p, \tau, t$. Then we say that $\mathscr{M}$ satisfies the condition $\left(C_{z}\right)$ if we have the following:

(1) for $z=\sigma: \sup \left\{|\mu|\left(Z_{n}\right): \mu \in \mathscr{M}\right\} \rightarrow 0$ whenever $Z_{n} \downarrow \emptyset,\left(Z_{n}\right) \subset \mathscr{Z}$;

(2) for $z=\infty$ : for every partition of unity $\left(u_{\alpha}\right)_{\alpha \in \mathscr{A}}$ for $X$ and every $\varepsilon>0$ there exists a finite set $\mathscr{A}_{\varepsilon}$ in $\mathscr{A}$ such that $\sup _{\mu \in \mathscr{M}} \int_{X}\left(1-\sum_{\alpha \in \mathscr{A}_{\varepsilon}} u_{\alpha}\right) d|\mu|<\varepsilon$;
(3) for $z=p$ : for every continuous function $f$ from $X$ onto a separable metric space $Y$ and every $\varepsilon>0$, there is a compact subset $K$ of $Y$ such that $\sup _{\mu \in \mathscr{M}}|\mu|(X)$ $\left.\bar{f}^{1}(K)\right) \leq \varepsilon$

(4) for $z=\tau: \sup \left\{|\mu|\left(Z_{\alpha}\right): \mu \in \mathscr{M}\right\} \rightarrow 0$ whenever $Z_{\alpha} \downarrow \emptyset,\left(Z_{\alpha}\right) \subset \mathscr{Z}$;

(5) for $z=t$ : for every $\varepsilon>0$ there exists a compact subset $K$ of $X$ such that $\sup \{|\mu|(Z): Z \in \mathscr{Z}, Z \subset X \backslash K\} \leq \varepsilon$ for each $\mu \in \mathscr{M}$.

The following lemmas will be useful.

Lemma 4. Assume that $\mathscr{M}$ is a subset of $M_{z}\left(X, E^{\prime}\right)$ and $\sup _{\mu \in \mathscr{M}}|\mu|(X)<\infty$, where $z=\sigma$ and $C_{b}(X) \otimes E$ is $\beta_{\sigma}$-dense in $C_{b}(X, E)$ (resp., $z=\infty ; z=p$ and $C_{b}(X) \otimes E$ is $\beta_{p}$-dense in $\left.C_{b}(X, E) ; z=\tau ; z=t\right)$. Then the following statements are equivalent.

(i) $\left\{\Phi_{\mu}: \mu \in \mathscr{M}\right\}$ is $\beta_{z}$-equicontinuous.

(ii) $\left\{\left|\Phi_{\mu}\right|: \mu \in \mathscr{M}\right\}$ is $\beta_{z}$-equicontinuous.

(iii) $\left\{\varphi_{|\mu|}: \mu \in \mathscr{M}\right\}$ is $\beta_{z}$-equicontinuous.

(iv) The condition $\left(C_{z}\right)$ holds.

Proof. (i) $\Leftrightarrow$ (ii) See [9, Lemma 2].

(ii) $\Leftrightarrow$ (iii) It follows from Theorem 3 .

(iii) $\Leftrightarrow$ (iv) See [4, Theorem 11.14] for $z=\sigma$; [28, Proposition 3.6] for $z=\infty$; [28, Proposition 2.6] for $z=p$; [4, Theorem 11.24] for $z=\tau$; and [28, Proposition 1.1] for $z=$ $t$.

Lemma 5. Assume that $z=\sigma$ and $C_{b}(X) \otimes E$ is $\beta_{\sigma}$-dense in $C_{b}(X, E)$ (resp., $z=\infty ; z=p$, and $C_{b}(X) \otimes E$ is $\beta_{p}$-dense in $\left.C_{b}(X, E) ; z=\tau ; z=t\right)$. Let $\mu \in M_{z}\left(X, E^{\prime}\right)$. Then for $A \in \mathscr{B}$ the following statements hold.

(i) A functional $\Phi_{A}: C_{r c}(X, E) \rightarrow \mathbb{R}$ defined by $\Phi_{A}(h)=$ $\int_{A} h d \mu$ is $\left.\beta_{z}\right|_{C_{r c}(X, E)}$-continuous and can by uniquely extended to a $\beta_{z}$-continuous linear functional $\overline{\Phi_{A}}$ : $C_{b}(X, E) \rightarrow \mathbb{R}$, and one will write the following:

$$
\int_{A} f d \mu:=\overline{\Phi_{A}}(f) \quad \text { for } f \in C_{b}(X, E) .
$$

(ii) $\left|\int_{A} f d \mu\right| \leq \int_{A} \tilde{f} d|\mu|$ for $f \in C_{b}(X, E)$.

Proof. (i) Assume that $\left(h_{\alpha}\right)$ is a net in $C_{r c}(X, E)$ such that $h_{\alpha} \rightarrow 0$ for $\beta_{z}$. Then

$$
\left|\Phi_{A}\left(h_{\alpha}\right)\right|=\left|\int_{A} h_{\alpha} d \mu\right| \leq \int_{A} \widetilde{h}_{\alpha} d|\mu| \leq \int_{X} \widetilde{h}_{\alpha} d|\mu| .
$$

Since $\tilde{h}_{\alpha} \rightarrow 0$ for $\beta_{z}$ in $C_{b}(X)$ and $|\mu| \in M_{z}(X)$, we obtain that $\Phi_{A}\left(h_{\alpha}\right) \rightarrow 0$; that is, $\Phi_{A}$ is $\left.\beta_{z}\right|_{C_{r c}(X, E)}$-continuous. Since $C_{r c}(X, E)$ is dense in $\left(C_{b}(X, E), \beta_{z}\right), \Phi_{A}$ can be uniquely extended to a $\beta_{z}$-continuous linear functional $\overline{\Phi_{A}}: C_{b}(X, E) \rightarrow \mathbb{R}($ see $[29$, Theorem 2.6]). 
(ii) Assume that $f \in C_{b}(X, E)$. Choose a net $\left(h_{\alpha}\right)$ in $C_{r c}(X, E)$ such that $h_{\alpha} \rightarrow f$ for $\beta_{z}$. Then $\widetilde{h}_{\alpha} \rightarrow \tilde{f}$ for $\beta_{z}$ in $C_{b}(X)$. Then

$$
\begin{aligned}
\left|\int_{A} \tilde{h}_{\alpha} d\right| \mu\left|-\int_{A} \tilde{f} d\right| \mu|| & \leq \int_{A}\left|\widetilde{h}_{\alpha}-\tilde{f}\right| d|\mu| \\
& \leq \int_{X}\left|\widetilde{h}_{\alpha}-\tilde{f}\right| d|\mu|,
\end{aligned}
$$

and hence $\int_{A} \tilde{f} d|\mu|=\lim _{\alpha} \int_{A} \widetilde{h}_{\alpha} d|\mu|$. Since $\int_{A} f d \mu=$ $\overline{\Phi_{A}}(f)=\lim _{\alpha} \int_{A} h_{\alpha} d \mu$, we get

$$
\begin{aligned}
\left|\int_{A} f d \mu\right| & =\lim _{\alpha}\left|\int_{A} h_{\alpha} d \mu\right| \\
& \leq \lim _{\alpha} \int_{A} \tilde{h}_{\alpha} d|\mu|=\int_{A} \tilde{f} d|\mu| .
\end{aligned}
$$

For $z=\sigma, \infty, p, \tau, t$ let us put

$$
\begin{aligned}
& M_{z}\left(X, \mathscr{L}\left(E, F^{\prime \prime}\right)\right) \\
&:=\left\{m \in M\left(X, \mathscr{L}\left(E, F^{\prime \prime}\right)\right): m_{y^{\prime}} \in M_{z}\left(X, E^{\prime}\right)\right. \\
&\left.\quad \text { for each } y^{\prime} \in F^{\prime}\right\} .
\end{aligned}
$$

Lemma 6. Assume that $z=\sigma$ and $C_{b}(X) \otimes E$ is $\beta_{\sigma}$-dense in $C_{b}(X, E)$ (resp., $z=\infty ; z=p$, and $C_{b}(X) \otimes E$ is $\beta_{p}$-dense in $\left.C_{b}(X, E) ; z=\tau ; z=t\right)$. Assume that $m \in M_{z}\left(X, \mathscr{L}\left(E, F^{\prime \prime}\right)\right)$ and the set $\left\{m_{y^{\prime}}: y^{\prime} \in F^{\prime}\right\}$ satisfies the condition $\left(C_{z}\right)$. Then for $A \in \mathscr{B}$ the following statements hold.

(i) An operator $S_{A}: C_{r c}(X, E) \rightarrow F^{\prime \prime}$ defined by $S_{A}(h)=$ $\int_{A} h d m$ is $\left(\left.\beta_{z}\right|_{C_{r c}(X, E)},\|\cdot\|_{F^{\prime \prime}}\right)$-continuous and can be uniquely extended to a $\left(\beta_{z},\|\cdot\|_{F^{\prime \prime}}\right)$-continuous linear operator $\overline{S_{A}}: C_{b}(X, E) \rightarrow F^{\prime \prime}$, and one will write the following.

$$
\int_{A} f d m:=\overline{S_{A}}(f) \text { for } f \in C_{b}(X, E) .
$$

(ii) For each $y^{\prime} \in F^{\prime},\left(\int_{A} f d m\right)\left(y^{\prime}\right)=\int_{A} f d m_{y^{\prime}}$ for $f \in$ $C_{b}(X, E)$.

Proof. (i) In view of Lemma 5 the set $\left\{\varphi_{\left|m_{y^{\prime}}\right|}: y^{\prime} \in B_{F^{\prime}}\right\}$ is $\beta_{z}$-equicontinuous in $C_{b}(X)_{\beta_{z}}^{\prime}$. Assume that $\left(h_{\alpha}\right)$ is a net in $C_{r c}(X, E)$ such that $h_{\alpha} \rightarrow 0$ for $\beta_{z}$. Let $\varepsilon>0$ be given. Then there exists a neighborhood $V_{\varepsilon}$ of 0 for $\beta_{z}$ in $C_{b}(X)$ such that $\sup _{y^{\prime} \in B_{F^{\prime}}}\left|\int_{X} u d\right| m_{y^{\prime}}|| \leq \varepsilon$ for $u \in V_{\varepsilon}$. Since $\widetilde{h}_{\alpha} \rightarrow 0$ for $\beta_{z}$ in $C_{b}(X)$, choose $\alpha_{\varepsilon}$ such that $h_{\alpha} \in V_{\varepsilon}$ for $\alpha \geq \alpha_{\varepsilon}$. Hence $\sup _{y^{\prime} \in B_{F^{\prime}}} \int_{X} \widetilde{h}_{\alpha} d\left|m_{y^{\prime}}\right| \leq \varepsilon$ for $\alpha \geq \alpha_{\varepsilon}$. It follows that, for $\alpha \geq \alpha_{\varepsilon}$ and each $y^{\prime} \in B_{F^{\prime}}$,

$$
\begin{aligned}
\left|\left(\int_{A} h_{\alpha} d m\right)\left(y^{\prime}\right)\right| & =\left|\int_{A} h_{\alpha} d m_{y^{\prime}}\right| \\
& \leq \int_{A} \widetilde{h}_{\alpha} d\left|m_{y^{\prime}}\right| \leq \int_{X} \widetilde{h}_{\alpha} d\left|m_{y^{\prime}}\right| \leq \varepsilon,
\end{aligned}
$$

and hence,

$$
\left\|S_{A}\left(h_{\alpha}\right)\right\|_{F^{\prime \prime}}=\sup \left\{\left|S_{A}\left(h_{\alpha}\right)\left(y^{\prime}\right)\right|: y^{\prime} \in B_{F^{\prime}}\right\} \leq \varepsilon .
$$

This means that $S_{A}: C_{r c}(X, E) \rightarrow F^{\prime \prime}$ is $\left(\left.\beta_{z}\right|_{C_{r c}(X, E)},\|\cdot\|_{F^{\prime \prime}}\right)$ continuous. Since $C_{r c}(X, E)$ is $\beta_{z}$-dense in $\left(C_{b}(X, E), \beta_{z}\right), S_{A}$ possesses a unique $\left(\beta_{z},\|\cdot\|_{F^{\prime \prime}}\right)$-continuous extension $\overline{S_{A}}$ : $C_{b}(X, E) \rightarrow F^{\prime \prime}$ (see [29, Theorem 2.6]). Let

$$
\int_{A} f d m:=\overline{S_{A}}(f) \text { for } f \in C_{b}(X, E) .
$$

(ii) Let $f \in C_{b}(X, E)$. Choose a net $\left(h_{\alpha}\right)$ in $C_{r c}(X, E)$ such that $h_{\alpha} \rightarrow f$ for $\beta_{z}$. By Lemma 5 and (7) for $y^{\prime} \in F^{\prime}$ we have

$$
\begin{aligned}
\left(\int_{A} f d m\right)\left(y^{\prime}\right) & =\left(\lim _{\alpha}\left(\int_{A} h_{\alpha} d m\right)\right)\left(y^{\prime}\right) \\
& =\lim _{\alpha}\left(\int_{A} h_{\alpha} d m_{y^{\prime}}\right)\left(y^{\prime}\right) \\
& =\lim _{\alpha} \int_{A} h_{\alpha} d m_{y^{\prime}}=\int_{A} f d m_{y^{\prime}} .
\end{aligned}
$$

Corollary 7. Assume that $z=\sigma$ and $C_{b}(X) \otimes E$ is $\beta_{\sigma}$-dense in $C_{b}(X, E)$ (resp., $z=\infty ; z=p$ and $C_{b}(X) \otimes E$ is $\beta_{p}$-dense in $\left.C_{b}(X, E) ; z=\tau ; z=t\right)$. Assume that $m \in M_{z}\left(X, \mathscr{L}\left(E, F^{\prime \prime}\right)\right)$ and the set $\left\{m_{y^{\prime}}: y^{\prime} \in B_{F^{\prime}}\right\}$ satisfies the condition $\left(C_{z}\right)$. Then for $A \in \mathscr{B}$ the following statements hold:

(a) $\left|m_{y^{\prime}}\right|(A)$

$$
\begin{aligned}
& =\sup \left\{\left|\int_{A} h d m_{y^{\prime}}\right|: h \in C_{b}(X) \otimes E,\|h\| \leq 1\right\} \\
& =\sup \left\{\left|\int_{A} f d m_{y^{\prime}}\right|: f \in C_{b}(X, E),\|f\| \leq 1\right\} .
\end{aligned}
$$

(b) $\widetilde{m}(A)$

$$
\begin{aligned}
& =\sup \left\{\left\|\int_{A} h d m\right\|_{F^{\prime \prime}}: h \in C_{b}(X) \otimes E,\|h\| \leq 1\right\} \\
& =\sup \left\{\left\|\int_{A} f d m\right\|_{F^{\prime \prime}}: f \in C_{b}(X, E),\|f\| \leq 1\right\} .
\end{aligned}
$$

In particular, if $U \in \mathscr{P}$, then

(c) $\left|m_{y^{\prime}}\right|(U)=\sup \left\{\left|\int_{U} h d m_{y^{\prime}}\right|: h \in C_{b}(X) \otimes E\right.$,

$\|h\| \leq 1, \operatorname{supp} h \subset U\}$ 
where the supremum is taken over all finite disjoint supported collections $\left\{u_{1}, \ldots, u_{n}\right\} \subset C_{b}(X)$ with $\left\|u_{i}\right\| \leq 1$ and supp $u_{i} \subset$ $U$ and $\left\{x_{1}, \ldots, x_{n}\right\} \subset B_{E}$. One has

(d) $\widetilde{m}(U)=\sup \left\{\left\|\int_{U} h d m\right\|_{F^{\prime \prime}}: h \in C_{b}(X) \otimes E\right.$,

$$
\begin{gathered}
\|h\| \leq 1, \operatorname{supp} h \subset U\} \\
=\sup \left\{\left\|\int_{U} f d m\right\|_{F^{\prime \prime}}: f \in C_{b}(X, E),\right. \\
\|f\| \leq 1, \operatorname{supp} f \subset U\} .
\end{gathered}
$$

Proof. Let $A \in \mathscr{B}$ and $y^{\prime} \in F^{\prime}$. Then by Lemma 5 for $f \in$ $C_{b}(X, E)$ with $\|f\| \leq 1$ we have

$$
\left|\int_{A} f d m_{y^{\prime}}\right| \leq \int_{A} \tilde{f} d\left|m_{y^{\prime}}\right| \leq\left|m_{y^{\prime}}\right|(A)
$$

On the other hand, let $\varepsilon>0$ be given. Then there exist a finite $\mathscr{B}$-partition $\left(A_{i}\right)_{i=1}^{n}$ of $A$ and $x_{i} \in B_{E}, i=1, \ldots, n$, such that

$$
\left|m_{y^{\prime}}\right|(A)-\frac{\varepsilon}{3} \leq\left|\sum_{i=1}^{n}\left(m\left(A_{i}\right)\left(x_{i}\right)\right)\left(y^{\prime}\right)\right|=\left|\sum_{i=1}^{n} m_{x_{i}, y^{\prime}}\left(A_{i}\right)\right| .
$$

By the regularity of $m_{x_{i}, y^{\prime}} \in M_{z}(X)$ for $i=1, \ldots, n$, we can choose $Z_{i} \in \mathscr{Z}, Z_{i} \subset A_{i}$ such that $\left|m_{x_{i}, y^{\prime}}\right|\left(A_{i} \backslash Z_{i}\right) \leq \varepsilon / 3 n$ for $i=1, \ldots, n$. Choose pairwise disjoint $V_{i} \in \mathscr{P}$ with $Z_{i} \subset V_{i}$ for $i=1, \ldots, n$ such that $\left|m_{x_{i}, y^{\prime}}\right|\left(V_{i} \backslash Z_{i}\right) \leq \varepsilon / 3 n$. Then for $i=1, \ldots, n$ we can choose $v_{i} \in C_{b}(X)$ with $0 \leq$ $v_{i} \leq \mathbb{1}_{X},\left.v_{i}\right|_{Z_{i}} \equiv 1$, and $\left.v_{i}\right|_{X \backslash V_{i}} \equiv 0$ (see [4, page 115]). Define $h_{o}=\sum_{i=1}^{n}\left(v_{i} \otimes x_{i}\right)$. Then $\left\|h_{o}\right\| \leq 1$ and $\int_{A} h_{o} d m_{y^{\prime}}=$ $\sum_{i=1}^{n} \int_{A} v_{i} d m_{x_{i}, y^{\prime}}=\sum_{i=1}^{n} \int_{V_{i} \cap A} v_{i} d m_{x_{i}, y^{\prime}}$. Hence we get

$$
\begin{aligned}
\left|m_{y^{\prime}}\right|(A)-\frac{\varepsilon}{3} \leq & \left|\sum_{i=1}^{n} m_{x_{i}, y^{\prime}}\left(A_{i}\right)-\sum_{i=1}^{n} m_{x_{i}, y^{\prime}}\left(Z_{i}\right)\right| \\
& +\left|\sum_{i=1}^{n} \int_{Z_{i}} v_{i} d m_{x_{i}, y^{\prime}}-\sum_{i=1}^{n} \int_{V_{i} \cap A} v_{i} d m_{x_{i}, y^{\prime}}\right| \\
& +\left|\int_{A} h_{o} d m_{y^{\prime}}\right| \\
\leq & \sum_{i=1}^{n}\left|m_{x_{i}, y^{\prime}}\right|\left(A_{i} \backslash Z_{i}\right)+\sum_{i=1}^{n}\left|m_{x_{i}, y^{\prime}}\right|\left(V_{i} \backslash Z_{i}\right) \\
& +\left|\int_{A} h_{o} d m_{y^{\prime}}\right| \\
\leq & \frac{\varepsilon}{3}+\frac{\varepsilon}{3}+\left|\int_{A} h_{o} d m_{y^{\prime}}\right|
\end{aligned}
$$

and hence $\left|m_{y^{\prime}}\right|(A) \leq\left|\int_{A} h_{o} d m_{y^{\prime}}\right|+\varepsilon$. Thus the proof of (a) is complete.
In view of (5), (a), and Lemma 6 we get

$$
\begin{aligned}
\widetilde{m}(A)= & \sup \left\{\left|m_{y^{\prime}}\right|(A): y^{\prime} \in B_{F^{\prime}}\right\} \\
= & \sup \left\{\left|\left(\int_{A} h d m\right)\left(y^{\prime}\right)\right|: h \in C_{b}(X) \otimes E,\right. \\
& \left.\|h\| \leq 1, y^{\prime} \in B_{F^{\prime}}\right\} \\
= & \sup \left\{\left|\left(\int_{A} f d m\right)\left(y^{\prime}\right)\right|: f \in C_{b}(X, E),\right. \\
= & \sup \left\{\left\|\left(\int_{A} h d m\right)\right\|_{F^{\prime \prime}}: h \in C_{b}(X) \otimes E,\|h\| \leq 1\right\} \\
= & \sup \left\{\left\|\left(\int_{A} f d m\right)\right\|_{F^{\prime \prime}}: f \in B_{F^{\prime}}\right\}
\end{aligned}
$$

that is, (b) holds.

Assume now that $U \in \mathscr{P}$. Let $U_{i}=V_{i} \cap U \in \mathscr{P}$ for $i=$ $1, \ldots, n$. Then $\left|m_{x_{i}, y^{\prime}}\right|\left(U_{i} \backslash Z_{i}\right) \leq\left|m_{x_{i}, y^{\prime}}\right|\left(V_{i} \backslash Z_{i}\right) \leq \varepsilon / 3 n$ for $i=$ $1, \ldots, n$. For $i=1, \ldots, n$ choose $u_{i} \in C_{b}(X)$ with $0 \leq u_{i} \leq \mathbb{1}_{X}$, $\left.u_{i}\right|_{Z_{i}} \equiv 1$, and $\left.u_{i}\right|_{X \backslash U_{i}} \equiv 0$. Let $h_{o}=\sum_{i=1}^{n}\left(u_{i} \otimes x_{i}\right)$. Then $\left\|h_{o}\right\| \leq 1$ and supp $h_{o} \subset U$; and hence by (a), $\left|m_{y^{\prime}}\right|(U) \leq\left|\int_{U} h_{o} d m_{y^{\prime}}\right|+$ $\varepsilon$. Note that $\int_{U} h_{o} d m_{y^{\prime}}=\sum_{i=1}^{n} \int_{X} u_{i} d m_{x_{i}, y^{\prime}}$, where supp $u_{i}$ are pairwise disjoint and supp $u_{i} \subset U$ for $i=1, \ldots, n$. Thus (c) holds.

Using (c) we easily show that (d) holds. Thus the proof is complete.

Definition 8. Let $T: C_{b}(X, E) \rightarrow F$ be a bounded linear operator. Then the measure $m \in M\left(X, \mathscr{L}\left(E, F^{\prime \prime}\right)\right)$ defined by

$$
\begin{array}{r}
m(A)(x):=\left(\left(\left.T\right|_{C_{r c}(X, E)}\right)^{\prime \prime} \circ \pi\right)\left(\mathbb{1}_{A} \otimes x\right) \\
\text { for } A \in \mathscr{B}, x \in E
\end{array}
$$

will be called a representing measure of $T$.

Now we state general Riesz representation theorems for continuous linear operators on $C_{b}(X, E)$, provided with the strict topologies $\beta_{z}$, where $z=\sigma, \infty, p, \tau, t$.

Theorem 9. Assume that $z=\sigma$ and $C_{b}(X) \otimes E$ is $\beta_{\sigma}$-dense in $C_{b}(X, E)$ (resp., $z=\infty ; z=p$, and $C_{b}(X) \otimes E$ is $\beta_{p}$-dense in $\left.C_{b}(X, E) ; z=\tau ; z=t\right)$.

(I) Let $T: C_{b}(X, E) \rightarrow F$ be a $\left(\beta_{z},\|\cdot\|_{F}\right)$-continuous linear operator and let $m \in M\left(X, \mathscr{L}\left(E, F^{\prime \prime}\right)\right)$ be its representing measure. Then the following statements hold.

(i) $m \in M_{z}\left(X, \mathscr{L}\left(E, F^{\prime \prime}\right)\right)$ and $\left\{m_{y^{\prime}}: y^{\prime} \in B_{F^{\prime}}\right\}$ satisfies the condition $\left(C_{z}\right)$.

(ii) For each $y^{\prime} \in F^{\prime}, y^{\prime}(T(f))=\int_{X} f d m_{y^{\prime}}$ for $f \in$ $C_{b}(X, E)$. 
(iii) For each $f \in C_{b}(X, E)$ and $A \in \mathscr{B}$ there exists a unique vector in $F^{\prime \prime}$, denoted by $\int_{A} f d m$, such that $\left(\int_{A} f d m\right)\left(y^{\prime}\right)=\int_{A} f d m_{y^{\prime}}$ for each $y^{\prime} \in F^{\prime}$.

(iv) For each $A \in \mathscr{B}$, the mapping $C_{b}(X, E) \ni f \mapsto$ $\int_{A} f d m \in F^{\prime \prime}$ is a $\left(\beta_{z},\|\cdot\|_{F^{\prime \prime}}\right)$-continuous linear operator.

(v) For $f \in C_{b}(X, E), \int_{X} f d m \in i_{F}(F)$ and $T(f)=$ $j_{F}\left(\int_{X} f d m\right)$.

(vi) $\|T\|=\widetilde{m}(X)$.

(II) Let $m \in M_{z}\left(X, \mathscr{L}\left(E, F^{\prime \prime}\right)\right)$ and let the set $\left\{m_{y^{\prime}}: y^{\prime} \in\right.$ $B_{F^{\prime}}$ satisfy the condition $\left(C_{z}\right)$. Then the statements (iii) and (iv) hold and for $f \in C_{b}(X, E), \int_{X} f d m \in i_{F}(F)$ and the mapping $T: C_{b}(X, E) \rightarrow F$ defined by $T(f):=j_{F}\left(\int_{X} f d m\right)$ is a $\left(\beta_{z},\|\cdot\|_{F}\right)$-continuous linear operator. Moreover, $m$ coincides with the representing measure of $T$ and the statements (ii) and (vi) hold.

Proof. (I) In view of (10) for each $y^{\prime} \in F^{\prime}, y^{\prime}(T(h))=$ $\int_{X} h d m_{y^{\prime}}$ for $h \in C_{r c}(X, E)$. By Theorem 3 for each $y^{\prime} \in F^{\prime}$ there exists a unique $\mu_{y^{\prime} \circ T} \in M_{z}\left(X, E^{\prime}\right)$ such that $\left(y^{\prime} \circ T\right)(f)=$ $\int_{X} f d \mu_{y^{\prime} \circ T}$ for $f \in C_{b}(X, E)$. It follows that, for each $y^{\prime} \in F^{\prime}$, $m_{y^{\prime}}=\mu_{y^{\prime} \circ T}$ (see [23, Theorem 2.5]) and this means that $m \in M_{z}\left(X, \mathscr{L}\left(E, F^{\prime \prime}\right)\right)$. Hence

$$
y^{\prime}(T(f))=\int_{X} f d m_{y^{\prime}} \text { for } f \in C_{b}(X, E) .
$$

Since $\left\{y^{\prime} \circ T: y^{\prime} \in B_{F^{\prime}}\right\}$ is $\beta_{z}$-equicontinuous in $C_{b}(X, E)_{\beta_{z}}^{\prime}$, by Lemma 4 the set $\left\{m_{y^{\prime}}: y^{\prime} \in B_{F^{\prime}}\right\}$ satisfies the condition $\left(C_{z}\right)$. Thus (i) and (ii) hold. In view of Lemma 6 , (iii) and (iv) are satisfied.

According to (9) for each $h \in C_{r c}(X, E), \int_{X} h d m \in i_{F}(F)$ and $T(h)=j_{F}\left(\int_{X} h d m\right)$. Hence by Lemma $6, \int_{X} f d m \in i_{F}(F)$. Let $f \in C_{b}(X, E)$. Choose a net $\left(h_{\alpha}\right)$ in $C_{r c}(X, E)$ such that $h_{\alpha} \rightarrow f$ for $\beta_{z}$. Hence

$$
\begin{aligned}
T(f) & =\lim _{\alpha} T\left(h_{\alpha}\right)=\lim _{\alpha} j_{F}\left(\int_{X} h_{\alpha} d m\right) \\
& =j_{F}\left(\lim _{\alpha} \int_{X} h_{\alpha} d m\right)=j_{F}\left(\int_{X} f d m\right) .
\end{aligned}
$$

Thus (v) holds. Using (v) and Corollary 7 we get $\|T\|=\widetilde{m}(X)$.

(II) By Lemma 6 the statements (iii) and (iv) are satisfied.

Now let $f \in C_{b}(X, E)$. Choose a net $\left(h_{\alpha}\right)$ in $C_{r c}(X, E)$ such that $h_{\alpha} \rightarrow f$ for $\beta_{z}$. Then by Lemma $6, \int_{X} f d m=\overline{S_{X}}(f)=$ $\lim _{\alpha} \int_{X} h_{\alpha} d m \in i_{F}(F)$ because $\int_{X} h_{\alpha} d m \in i_{F}(F)$, and it follows that $T\left(=j_{F} \circ \overline{S_{X}}\right)$ is $\left(\beta_{z},\|\cdot\|_{F}\right)$-continuous.
Let $m_{o} \in M\left(X, \mathscr{L}\left(E, F^{\prime \prime}\right)\right)$ stand for the representing measure of $T$. Note that, for $A \in \mathscr{B}, x \in E$, and $y^{\prime} \in F^{\prime}$ we have

$$
\begin{aligned}
\left(m_{o}(A)(x)\right)\left(y^{\prime}\right) & =\left(\left(\left(\left.T\right|_{C_{r c}(X, E)}\right)^{\prime \prime} \circ \pi\right)\left(\mathbb{1}_{A} \otimes x\right)\right)\left(y^{\prime}\right) \\
& =\pi\left(\mathbb{1}_{A} \otimes x\right)\left(\left(\left.T\right|_{C_{r c}(X, E)}\right)^{\prime}\left(y^{\prime}\right)\right) \\
& =\pi\left(\mathbb{1}_{A} \otimes x\right)\left(y^{\prime} \circ\left(\left.T\right|_{C_{r c}(X, E)}\right)\right) \\
& =\int_{X}\left(\mathbb{1}_{A} \otimes x\right) d m_{y^{\prime}}=\int_{X} \mathbb{1}_{A} d m_{x, y^{\prime}} \\
& =(m(A)(x))\left(y^{\prime}\right) ;
\end{aligned}
$$

that is, $m_{o}=m$. By the first part of the proof (ii) and (vi) hold. Thus the proof is complete.

Following $[14,27]$ by $M_{\sigma}(\mathscr{B} a)$ we denote the space of all bounded countably additive, real-valued, regular (with respect to zero sets) measures on $\mathscr{B} a$.

We define $M_{\sigma}\left(\mathscr{B} a, E^{\prime}\right)$ to be the set of all measures $\mu$ : $\mathscr{B} a \rightarrow E^{\prime}$ such that the following two conditions are satisfied.

(i) For each $x \in E$, the function $\mu_{x}: \mathscr{B} a \rightarrow \mathbb{R}$, defined by $\mu_{x}(A)=\mu(A)(x)$ for $A \in \mathscr{B} a$, belongs to $M_{\sigma}(\mathscr{B} a)$.

(ii) $|\mu|(X)<\infty$, where for each $A \in \mathscr{B} a$, we define $|\mu|(A)=\sup \left|\sum \mu\left(A_{i}\right)\left(x_{i}\right)\right|$, where the supremum is taken over all finite $\mathscr{B} a$-partitions $\left(A_{i}\right)$ of $A$ and all finite collections $x_{i} \in B_{E}$.

It is known that if $\mu \in M_{\sigma}\left(\mathscr{B} a, E^{\prime}\right)$, then $|\mu| \in M_{\sigma}(\mathscr{B} a)$ (see [27, Lemma 2.1]).

The following result will be of importance (see [27, Theorem 2.5]).

Theorem 10. Let $\mu \in M_{\sigma}\left(X, E^{\prime}\right)$. Then $\mu$ possesses a unique extension $\bar{\mu} \in M_{\sigma}\left(\mathscr{B} a, E^{\prime}\right)$ and $|\bar{\mu}|(X)=|\mu|(X)$.

Arguing as in the proof of Lemma 6 we can obtain the following lemma.

Lemma 11. Assume that $C_{b}(X) \otimes E$ is $\beta_{\sigma}$-dense in $C_{b}(X, E)$ and $\mu \in M_{\sigma}\left(X, E^{\prime}\right)$. Then for $A \in \mathscr{B}$ a the following statements hold.

(i) A functional $\Phi_{A}: C_{r c}(X, E) \rightarrow \mathbb{R}$ defined by $\Phi_{A}(h)=$ $\int_{A} h d \bar{\mu}$ is $\left.\beta_{\sigma}\right|_{C_{r c}(X, E)}$-continuous and can be uniquely extended to a $\beta_{\sigma}$-continuous linear functional $\overline{\Phi_{A}}$ : $C_{b}(X, E) \rightarrow \mathbb{R}$, and one will write the following:

$$
\int_{A} f d \bar{\mu}:=\overline{\Phi_{A}}(f) \text { for } f \in C_{b}(X, E) .
$$

(ii) For $f \in C_{b}(X, E),\left|\int_{A} f d \bar{\mu}\right| \leq \int_{A} \tilde{f} d|\bar{\mu}|$.

By $M_{\sigma}(X, \mathscr{L}(E, F))$ we will denote the space of all operator measures $m: \mathscr{B} \rightarrow \mathscr{L}(E, F)$ such that $\widetilde{m}(X)<\infty$ and 
$m_{y^{\prime}} \in M_{\sigma}\left(X, E^{\prime}\right)$ for each $y^{\prime} \in F^{\prime}$. By $M_{\sigma}(\mathscr{B} a, \mathscr{L}(E, F))$ we will denote the space of all operator measures $m: \mathscr{B} a \rightarrow$ $\mathscr{L}(E, F)$ with $\widetilde{m}(X)<\infty$ such that $m_{y^{\prime}} \in M_{\sigma}\left(\mathscr{B} a, E^{\prime}\right)$ for each $y^{\prime} \in F^{\prime}$.

Remark 12. Note that in view of the Orlicz-Pettis theorem every $m \in M_{\sigma}(\mathscr{B} a, \mathscr{L}(E, F))$ is countably additive in the strong operator topology; that is, for each $x \in E$, the measure $m_{x}: \mathscr{B} a \rightarrow F$ defined by $m_{x}(A):=m(A)(x)$ for $A \in \mathscr{B} a$ is countably additive. Moreover, in view of [30, Theorem 2] for each $x \in E, m_{x}$ is inner regular by zero sets and outer regular by cozero sets; that is, for each $A \in \mathscr{B} a$ and $\varepsilon>0$ there exist $Z \in \mathscr{Z}$ with $Z \subset A$ and $P \in \mathscr{P}$ with $A \subset \mathscr{P}$ such that $\left\|m_{x}\right\|(A \backslash Z) \leq \varepsilon$ and $\left\|m_{x}\right\|(P \backslash A) \leq \varepsilon,\left(\left\|m_{x}\right\|(A)\right.$ denotes the semivariation of $m_{x}$ on $A \in \mathscr{B} a$ ).

According to [14, Theorem 7] we have the following theorem.

Theorem 13. Assume that $m \in M_{\sigma}(X, \mathscr{L}(E, F))$ and $\{m(A)(x): A \in \mathscr{B}\}$ is a relatively weakly compact subset of $F$ for each $x \in E$. Then $m$ possesses a unique extension $\bar{m} \epsilon$ $M_{\sigma}(\mathscr{B} a, \mathscr{L}(E, F))$ such that $\widetilde{\bar{m}}(X)=\widetilde{m}(X)$.

For a linear operator $T: C_{b}(X, E) \rightarrow F$ and $x \in E$ let $T_{x}(u):=T(u \otimes x)$ for $u \in C_{b}(X)$. For $m \in M_{\sigma}\left(\mathscr{B}, \mathscr{L}\left(E, F^{\prime \prime}\right)\right)$ and $x \in E$ let $m_{x}(A):=m(A)(x)$ for $A \in \mathscr{B}$.

Theorem 14. Assume that $C_{b}(X) \otimes E$ is $\beta_{\sigma}$-dense in $C_{b}(X, E)$.

(I) Let $T: C_{b}(X, E) \rightarrow F$ be $a\left(\beta_{\sigma},\|\cdot\|_{F}\right)$-continuous linear operator such that $T_{x}: C_{b}(X) \rightarrow F$ is weakly compact for each $x \in E$, and let $m \in M\left(X, \mathscr{L}\left(E, F^{\prime \prime}\right)\right)$ be the representing measure of $T$. Then the following statements hold.

(i) $m \in M_{\sigma}\left(X, \mathscr{L}\left(E, F^{\prime \prime}\right)\right)$ and $\widetilde{m}\left(Z_{n}\right) \rightarrow 0$ whenever $Z_{n} \downarrow \emptyset,\left(Z_{n}\right) \subset \mathscr{Z}$.

(ii) $m(A)(x) \in i_{F}(F)$, for each $A \in \mathscr{B}, x \in E$, and the measure $m_{F}: \mathscr{B} \rightarrow \mathscr{L}(E, F)$, defined by $m_{F}(A)(x):=j_{F}(m(A)(x))$ for $A \in \mathscr{B}, x \in$ $E$, belongs to $M_{\sigma}(X, \mathscr{L}(E, F))$ and possesses a unique extension $\bar{m} \in M_{\sigma}(\mathscr{B} a, \mathscr{L}(E, F))$ with $\widetilde{\bar{m}}(X)=\widetilde{m}(X)$ which is countably additive both in the strong operator topology and in the weak star operator topology. Moreover, $\bar{m}_{y^{\prime}}=\bar{m}_{y^{\prime}}$ for $y^{\prime} \in F^{\prime}$.

(iii) For every $f \in C_{b}(X, E)$ and $A \in \mathscr{B}$ a there exists a unique vector in $F$, denoted by $\int_{A} f d \bar{m}$, such that, for each $y^{\prime} \in F^{\prime}, y^{\prime}\left(\int_{A} f d \bar{m}\right)=\int_{A} f d \bar{m}_{y^{\prime}}$.

(iv) For each $A \in \mathscr{B} a$, the mapping $T_{A}: C_{b}(X, E) \rightarrow$ $F$ defined by $T_{A}(f)=\int_{A} f d \bar{m}$ is a $\left(\beta_{\sigma},\|\cdot\|_{F}\right)$ continuous linear operator.

(v) $T(f)=T_{X}(f)=\int_{X} f d \bar{m}$ for $f \in C_{b}(X, E)$.

(II) Let $m \in M_{\sigma}\left(X, \mathscr{L}\left(E, F^{\prime \prime}\right)\right)$ be such that $\widetilde{m}\left(Z_{n}\right) \rightarrow 0$ whenever $Z_{n} \downarrow \emptyset,\left(Z_{n}\right) \subset \mathscr{Z}$ and for each $x \in E$, let $m_{x}: \mathscr{B} \rightarrow F^{\prime \prime}$ be strongly bounded. Then the operator
$T: C_{b}(X, E) \rightarrow F$ defined by $T(f)=j_{F}\left(\int_{X} f d m\right)$ is $\left(\beta_{\sigma},\|\cdot\|_{F}\right)$-continuous and $T_{x}: C_{b}(X) \rightarrow F$ is weakly compact for each $x \in E$, and the statements $(i i)-(v)$ hold.

Proof. (I) (i) It follows from Theorem 9.

(ii) In view of Theorem $2 m(A)(x) \in i_{F}(F)$ for $A \in \mathscr{B}$, $x \in E$, and $\left\{m_{F}(A)(x): A \in \mathscr{B}\right\}$ is a relatively weakly compact in $F$ for each $x \in E$. Since $m_{F} \in M_{\sigma}(X, \mathscr{L}(E, F))$, by Theorem $13 m_{F}$ possesses a unique extension $\bar{m} \epsilon$ $M_{\sigma}(\mathscr{B} a, \mathscr{L}(E, F))$ with $\widetilde{\bar{m}}(X)=\widetilde{m}(X)$. By the Orlicz-Pettis theorem $\bar{m}$ is countably additive in the strong operator topology. Moreover, since, for each $y^{\prime} \in F^{\prime},\left|\bar{m}_{y^{\prime}}\right| \epsilon$ $M_{\sigma}(\mathscr{B} a)=c a(\mathscr{B} a)$, we obtain that $\bar{m}_{y^{\prime}} \in c a\left(\mathscr{B} a, E^{\prime}\right)$. This means that $\bar{m}: \mathscr{B} a \rightarrow \mathscr{L}(E, F)$ is countably additive in the weak star operator topology.

Let $y^{\prime} \in F^{\prime}$. Then for $A \in \mathscr{B}$ and $x \in E$ we have $\bar{m}_{y^{\prime}}(A)(x)=m_{y^{\prime}}(A)(x)$, and by Theorem $10, \bar{m}_{y^{\prime}}=\overline{m_{y^{\prime}}}$.

(iii) For $A \in \mathscr{B} a$ let $S_{A}(h):=\int_{A} f d \bar{m}$ for $h \in C_{r c}(X, E)$. Proceeding as in the proof of Lemma 6 we can show that $S_{A}: C_{r c}(X, E) \rightarrow F$ is a $\left(\left.\beta_{\sigma}\right|_{C_{r c}(X, E)},\|\cdot\|_{F}\right)$-continuous linear operator, and hence $S_{A}$ possesses a unique $\left(\beta_{\sigma},\|\cdot\|_{F}\right)$ continuous linear extension $T_{A}: C_{b}(X, E) \rightarrow F$ (see [29, Theorem 2.6]). Let us write the following:

$$
\int_{A} f d \bar{m}:=T_{A}(f) \quad \text { for } f \in C_{b}(X, E) .
$$

Let $f \in C_{b}(X, E)$. Choose a net $\left(h_{\alpha}\right)$ in $C_{r c}(X, E)$ such that $h_{\alpha} \rightarrow f$ for $\beta_{\sigma}$. For each $y^{\prime} \in F^{\prime}, \bar{m}_{y^{\prime}}=\bar{m}_{y^{\prime}}$ (see (i)) and by Lemma 11 we have

$$
\begin{aligned}
y^{\prime}\left(\int_{A} f d \bar{m}\right) & =y^{\prime}\left(\lim _{\alpha} \int_{A} h_{\alpha} d \bar{m}\right)=\lim _{\alpha}\left(y^{\prime}\left(\int_{A} h_{\alpha} d \bar{m}\right)\right) \\
& =\lim _{\alpha} \int_{A} h_{\alpha} d \bar{m}_{y^{\prime}}=\lim _{\alpha} \int_{A} h_{\alpha} d \overline{m_{y^{\prime}}} \\
& =\int_{A} f d \bar{m}_{y^{\prime}}=\int_{A} f d \bar{m}_{y^{\prime}} .
\end{aligned}
$$

(iv) It follows from the proof of (iii).

(v) Let $f \in C_{b}(X, E)$. In view of Theorem 9 , for each $y^{\prime} \epsilon$ $F^{\prime}, y^{\prime}(T(f))=\int_{X} f d m_{y^{\prime}}$. On the other hand by (ii) for $y^{\prime} \in$ $F^{\prime}$ we have $y^{\prime}\left(\int_{X} f d \bar{m}\right)=\int_{X} f d \bar{m}_{y^{\prime}}=\int_{X} f d m_{y^{\prime}}$. It follows that $T(f)=\int_{X} f d \bar{m}$.

(II) Since $\left\{m_{y^{\prime}}: y^{\prime} \in B_{F^{\prime}}\right\}$ satisfies the condition $\left(C_{\sigma}\right)$, by Theorem 9 for $f \in C_{b}(X, E), \int_{X} f d m \in i_{F}(F)$ and the mapping $T: C_{b}(X, E) \rightarrow F$ defined by $T(f):=$ $j_{F}\left(\int_{X} f d m\right)$ is a $\left(\beta_{\sigma},\|\cdot\|_{F}\right)$-continuous linear operator, and $m$ coincides with the representing measure of $T$. Hence in view of Theorem $2 T_{x}: C_{b}(X) \rightarrow F$ is a weakly compact operator. Thus by the first part of the proof the statements (ii) $-(\mathrm{v})$ are satisfied.

\section{Strongly Bounded Operators on $C_{b}(X, E)$}

Definition 15. A bounded linear operator $T: C_{b}(X, E) \rightarrow$ $F$ is said to be strongly bounded if its representing measure 
$m \in M\left(X, \mathscr{L}\left(E, F^{\prime \prime}\right)\right)$ is strongly bounded; that is, $\widetilde{m}\left(A_{n}\right) \rightarrow$ 0 whenever $\left(A_{n}\right)$ is a pairwise disjoint sequence in $\mathscr{B}$.

Note that $m \in M\left(X, \mathscr{L}\left(E, F^{\prime \prime}\right)\right)$ is strongly bounded if and only if the family $\left\{\left|m_{y^{\prime}}\right|: y^{\prime} \in B_{F^{\prime}}\right\}$ is uniformly strongly additive.

Now we are ready to state our main results that extend some classical results of Lewis (see [20, Theorem 5], [31, Lemma 1]) and Brooks and Lewis (see [22, Theorem 2.1], [21, Theorem 5.2]) concerning operators on the spaces $C(X, E)$ and $C_{o}(X, E)$, where $X$ is a compact or a locally compact space, respectively.

Theorem 16. Assume that $C_{b}(X) \otimes E$ is $\beta_{\sigma}$-dense in $C_{b}(X, E)$. Let $T: C_{b}(X, E) \rightarrow F$ be a $\left(\beta_{\sigma},\|\cdot\|_{F}\right)$-continuous linear operator and let $m \in M\left(X, \mathscr{L}\left(E, F^{\prime \prime}\right)\right)$ be its representing measure. Then $m \in M_{\sigma}\left(X, \mathscr{L}\left(E, F^{\prime \prime}\right)\right)$ and the following statements are equivalent.

(i) $T$ is strongly bounded.

(ii) $\sup \left\{\left|\overline{m_{y^{\prime}}}\right|\left(A_{n}\right): y^{\prime} \in B_{F^{\prime}}\right\} \rightarrow 0$ whenever $A_{n} \downarrow \emptyset$, $\left(A_{n}\right) \subset \mathscr{B} a$ (here $\overline{m_{y^{\prime}}} \in M_{\sigma}\left(\mathscr{B} a, E^{\prime}\right)$ denotes the unique extension of $\left.m_{y^{\prime}} \in M_{\sigma}\left(X, E^{\prime}\right)\right)$.

(iii) If $\left(A_{n}\right)$ is a sequence in $\mathscr{B}$ a such that $A_{n} \downarrow \emptyset$, then there exists a nested sequence $\left(U_{n}\right)$ in $\mathscr{P}$ such that $A_{n} \subset U_{n}$ for $n \in \mathbb{N}$ and $\sup \left\{\|T(f)\|_{F}: f \in C_{b}(X, E),\|f\| \leq 1\right.$ and $\left.\operatorname{supp} f \subset U_{n}\right\} \rightarrow 0$.

Proof. In view of Theorem $9 m \in M_{\sigma}\left(X, \mathscr{L}\left(E, F^{\prime \prime}\right)\right)$.

(i) $\Rightarrow$ (ii) Assume that $T$ is strongly bounded. Since the family $\left\{\left|m_{y^{\prime}}\right|: y^{\prime} \in B_{F^{\prime}}\right\}$ is uniformly strongly additive, according to [25, Lemma 1, page 26] the family $\left\{\left|\overline{m_{y^{\prime}}}\right|: y^{\prime} \in\right.$ $\left.B_{F^{\prime}}\right\}$ is uniformly countably additive (see Theorem 16).

(ii) $\Rightarrow$ (i) It follows from [25, Lemma 1 , page 26].

(ii) $\Rightarrow$ (iii) Assume that (ii) holds and $\left(A_{n}\right)$ is a sequence in $\mathscr{B} a$ such that $A_{n} \downarrow \emptyset$. Then there exists $\lambda \in c a(\mathscr{B} a)^{+}$such that $\left\{\left|\overline{m_{y^{\prime}}}\right|: y^{\prime} \in B_{F^{\prime}}\right\}$ is uniformly $\lambda$-continuous (see [25, Theorem 4, pages 11-12]). Let $\varepsilon>0$ be given. Hence there exists $\delta>0$ such that $\sup \left\{\left|\overline{m_{y^{\prime}}}\right|(A): y^{\prime} \in B_{F^{\prime}}\right\} \leq \varepsilon / 2$ whenever $\lambda(A) \leq \delta$ and $A \in \mathscr{B} a$. Since $\lambda$ is zero-set regular, there exists a nested sequence $\left(U_{n}\right)$ in $\mathscr{P}$ so that $A_{n} \subset U_{n}$ and $\lambda\left(U_{n} \backslash A_{n}\right) \leq \delta$ for $n \in \mathbb{N}$. Hence $\sup \left\{\left|\overline{m_{y^{\prime}}}\right|\left(U_{n} \backslash A_{n}\right): y^{\prime} \epsilon\right.$ $\left.B_{F^{\prime}}\right\} \leq \varepsilon / 2$ for $n \in \mathbb{N}$. In view of (ii) there exists $n_{\varepsilon} \in \mathbb{N}$ such that $\sup \left\{\left|\overline{m_{y^{\prime}}}\right|\left(A_{n}\right): y^{\prime} \in B_{F^{\prime}}\right\} \leq \varepsilon / 2$ for $n \geq n_{\varepsilon}$. Hence $\sup \left\{\left|m_{y^{\prime}}\right|\left(U_{n}\right): y^{\prime} \in B_{F^{\prime}}\right\} \leq \varepsilon$ for $n \geq n_{\varepsilon}$; that is, $\sup \left\{\left|m_{y^{\prime}}\right|\left(U_{n}\right): y^{\prime} \in B_{F^{\prime}}\right\} \rightarrow 0$.

Let $f \in C_{b}(X, E),\|f\| \leq 1$, and supp $f \subset U_{n}$. Then by Theorem 9 we have

$$
\begin{aligned}
\|T(f)\|_{F} & =\sup \left\{\left|\int_{X} f d m_{y^{\prime}}\right|: y^{\prime} \in B_{F^{\prime}}\right\} \\
& \leq \sup \left\{\int_{X} \tilde{f} d\left|m_{y^{\prime}}\right|: y^{\prime} \in B_{F^{\prime}}\right\} \\
& \leq \sup \left\{\left|m_{y^{\prime}}\right|\left(U_{n}\right): y^{\prime} \in B_{F^{\prime}}\right\} .
\end{aligned}
$$

It follows that $\sup \left\{\|T(f)\|_{F}: f \in C_{b}(X, E),\|f\| \leq 1\right.$, supp $\left.f \subset U_{n}\right\} \rightarrow 0$. (iii) $\Rightarrow$ (ii) Assume that (iii) holds and $A_{n} \downarrow \emptyset,\left(A_{n}\right) \subset \mathscr{B} a$. Then there exists a nested sequence $\left(U_{n}\right)$ in $\mathscr{P}$ such that $A_{n} \subset$ $U_{n}$ for $n \in \mathbb{N}$ and

$$
\begin{aligned}
& \sup \left\{\|T(f)\|_{F}: f \in C_{b}(X, E),\|f\| \leq 1, \operatorname{supp} f_{n} \subset U_{n}\right\} \\
& \quad \longrightarrow 0 .
\end{aligned}
$$

Assume that (ii) does not hold. Then there exist $\varepsilon>0$ and $n_{\varepsilon} \in$ $\mathbb{N}$ such that $\sup \left\{\left|\overline{m_{y^{\prime}}}\right|\left(A_{n_{\varepsilon}}\right): y^{\prime} \in B_{F^{\prime}}\right\} \geq \varepsilon$ and $\|T(f)\|_{F} \leq$ $(1 / 8) \varepsilon$ whenever $f \in C_{b}(X, E),\|f\| \leq 1$, and supp $f \subset U_{n_{\varepsilon}}$. It follows that there exists $y_{o}^{\prime} \in B_{F^{\prime}}$ such that $\left|\overline{m_{y^{\prime}}}\right|\left(A_{n_{\varepsilon}}\right) \geq \varepsilon$. Hence there exist a finite $\mathscr{B} a$-partition $\left(B_{i}\right)_{i=1}^{k}$ of $A_{n_{\varepsilon}}$ and $x_{i} \in$ $B_{E}, i=1, \ldots, k$, such that

$$
\left|\overline{m_{y_{o}^{\prime}}}\right|\left(A_{n_{\varepsilon}}\right)-\frac{\varepsilon}{4} \leq\left|\sum_{i=1}^{k} \overline{m_{y_{o}^{\prime}}}\left(B_{i}\right)\left(x_{i}\right)\right|=\left|\sum_{i=1}^{k}\left(\overline{m_{y_{o}^{\prime}}}\right)_{x_{i}}\left(B_{i}\right)\right| .
$$

Since $\left|\left(\overline{m_{y_{o}^{\prime}}}\right)_{x_{i}}\right| \in M_{\sigma}(\mathscr{B} a)$ is zero-set regular (see [4, page 118]), we can choose $Z_{i} \in \mathscr{Z}, Z_{i} \subset B_{i}$, such that $\left|\left(\overline{m_{y_{o}^{\prime}}}\right)_{x_{i}}\right|\left(B_{i} \mid\right.$ $\left.Z_{i}\right) \leq \varepsilon / 4 k$ for $i=1, \ldots, k$. Choose pairwise disjoint $V_{i} \in \mathscr{P}$ with $Z_{i} \subset V_{i}$ for $i=1, \ldots, k$ such that $\left|m_{x_{i}, y_{o}^{\prime}}\right|\left(V_{i} \backslash Z_{i}\right) \leq \varepsilon / 4 k$. Let $U_{i}=V_{i} \cap U_{n_{\varepsilon}}$ for $i=1, \ldots, k$. Then $U_{i} \in \mathscr{P}$ and $\left|m_{x_{i}, y_{o}^{\prime}}\right|\left(U_{i} \mid\right.$ $\left.Z_{i}\right) \leq \varepsilon / 4 k$ for $i=1, \ldots, k$. For $i=1, \ldots, k$ choose $u_{i} \in C_{b}(X)$ such that $0 \leq u_{i} \leq \mathbb{1}_{X},\left.u_{i}\right|_{Z_{i}} \equiv 0$, and $\left.u_{i}\right|_{X \backslash U_{i}} \equiv 0$ (see [4, page $115])$. Let $h_{o}=\sum_{i=1}^{k}\left(u_{i} \otimes x_{i}\right)$. Then $\left\|h_{o}\right\| \leq 1$, supp $h_{o} \subset U_{n_{\varepsilon}}$, and

$$
\int_{U_{n_{\varepsilon}}} h_{o} d m_{y_{o}^{\prime}}=\sum_{i=1}^{k} \int_{U_{i}} u_{i} d m_{x_{i}, y_{o}^{\prime}}
$$

Hence we get

$$
\begin{aligned}
\left|\overline{m_{y_{o}^{\prime}}}\right| & \left(A_{n_{\varepsilon}}\right)-\frac{\varepsilon}{4} \\
\leq & \left|\sum_{i=1}^{k}\left(\overline{m_{y_{o}^{\prime}}}\right)_{x_{i}}\left(B_{i}\right)-\sum_{i=1}^{k}\left(\overline{m_{y_{o}^{\prime}}}\right)_{x_{i}}\left(Z_{i}\right)\right| \\
& +\left|\sum_{i=1}^{k} \int_{Z_{i}} u_{i} d m_{x_{i}, y_{o}^{\prime}}-\sum_{i=1}^{k} \int_{U_{i}} u_{i} d m_{x_{i}, y_{o}^{\prime}}\right| \\
& +\left|\int_{U_{n_{\varepsilon}}} h_{o} d m_{y_{o}^{\prime}}\right| \\
\leq & \sum_{i=1}^{k}\left|\left(\overline{m_{y_{o}^{\prime}}}\right)_{x_{i}}\right|\left(B_{i} \backslash Z_{i}\right)+\sum_{k=1}^{k}\left|m_{x_{i}, y_{o}^{\prime}}\right|\left(U_{i} \backslash Z_{i}\right) \\
& +\left|\int_{U_{n_{\varepsilon}}} h_{o} d m_{y_{o}^{\prime}}\right| \\
\leq & \frac{\varepsilon}{4}+\frac{\varepsilon}{4}+\left|\int_{U_{n_{\varepsilon}}} h_{o} d m_{y_{o}^{\prime}}\right| \cdot
\end{aligned}
$$


Hence

$$
\begin{gathered}
\left|\int_{U_{n_{\varepsilon}}} h_{o} d m_{y_{o}^{\prime}}\right| \geq\left|\overline{m_{y_{o}^{\prime}}}\right|\left(A_{n_{\varepsilon}}\right)-\frac{3}{4} \varepsilon \geq \frac{1}{4} \varepsilon, \\
\left\|T\left(h_{o}\right)\right\|_{F} \geq\left|y_{o}^{\prime}\left(T\left(h_{o}\right)\right)\right|=\left|\int_{X} h_{o} d m_{y_{o}^{\prime}}\right| \\
=\left|\int_{U_{n_{\varepsilon}}} h_{o} d m_{y_{o}^{\prime}}\right| \geq \frac{1}{4} \varepsilon .
\end{gathered}
$$

Thus we get a contradiction to $\left\|T\left(h_{o}\right)\right\|_{F} \leq(1 / 8) \varepsilon$.

Thus the proof is complete.

Theorem 17. Assume that $C_{b}(X) \otimes E$ is $\beta_{\sigma}$-dense in $C_{b}(X, E)$. Let $T: C_{b}(X, E) \rightarrow F$ be a $\left(\beta_{\sigma},\|\cdot\|_{F}\right)$-continuous and strongly bounded operator and let $m \in M\left(X, \mathscr{L}\left(E, F^{\prime \prime}\right)\right)$ be its representing measure. Then the following statements hold.

(i) $m \in M_{\sigma}\left(X, \mathscr{L}\left(E, F^{\prime \prime}\right)\right)$ and $m(A)(x) \in i_{F}(F)$ for $A \in \mathscr{B}, x \in E$, and the measure $m_{F}: \mathscr{B} \rightarrow \mathscr{L}(E, F)$, defined by $m_{F}(A)(x):=j_{F}(m(A)(x))$ for $A \in \mathscr{B}$, $x \in E$, belongs to $M_{\sigma}(X, \mathscr{L}(E, F))$ and possesses a unique extension $\bar{m} \in M_{\sigma}(\mathscr{B} a, \mathscr{L}(E, F))$ with $\widetilde{\bar{m}}(X)=$ $\widetilde{m}_{F}(X)=\widetilde{m}(X)$ which is variationally semiregular; that is, $\widetilde{\bar{m}}\left(A_{n}\right) \rightarrow 0$ whenever $A_{n} \downarrow \emptyset,\left(A_{n}\right) \subset \mathscr{B} a$.

(ii) For every $f \in C_{b}(X, E)$ and $A \in \mathscr{B}$ a there exists a unique vector in $F$, denoted by $\int_{A} f d \bar{m}$, such that, for each $y^{\prime} \in F^{\prime}, y^{\prime}\left(\int_{A} f d \bar{m}\right)=\int_{A} f d \bar{m}_{y^{\prime}}$.

(iii) For each $A \in \mathscr{B} a, \int_{A} f_{n} d \bar{m} \rightarrow 0$ whenever $\left(f_{n}\right)$ is a uniformly bounded sequence in $C_{b}(X, E)$ such that $f_{n}(t) \rightarrow 0$ for $t \in X$.

(iv) $T(f)=\int_{X} f d \bar{m}$ for $f \in C_{b}(X, E)$.

(v) $T\left(f_{n}\right) \rightarrow 0$ whenever $\left(f_{n}\right)$ is a uniformly bounded sequence in $C_{b}(X, E)$ such that $f_{n}(t) \rightarrow 0$ for $t \in X$.

Proof. (i) Note that, for $x \in E,\left\|m_{x}(A)\right\|_{F^{\prime \prime}} \leq \widetilde{m}(A)\|x\|_{E}$ for $A \in \mathscr{B}$. Hence $m_{x}: \mathscr{B} \rightarrow F^{\prime \prime}$ is strongly bounded, and by Theorems 2 and $14 m(A)(x) \in i_{F}(F)$ and $m_{F}$ possesses a unique extension $\bar{m} \in M_{\sigma}(\mathscr{B} a, \mathscr{L}(E, F))$ with $\widetilde{\bar{m}}(X)=$ $\widetilde{m}_{F}(X)=\widetilde{m}(X)$. Since $\bar{m}_{y^{\prime}}=\overline{m_{y^{\prime}}}$ for $y^{\prime} \in F^{\prime}$, by Theorem 16 we have $\widetilde{\bar{m}}\left(A_{n}\right)=\sup \left\{\left|\bar{m}_{y^{\prime}}\right|\left(A_{n}\right): y^{\prime} \in B_{F^{\prime}}\right\} \rightarrow 0$ whenever $A_{n} \downarrow \emptyset,\left(A_{n}\right) \subset \mathscr{B} a$.

(ii) It follows from Theorem 14 because for each $x \in E$, $T_{x}: C_{c}(X) \rightarrow F$ is weakly compact (see Theorem 2 ).

(iii) In view of (i) there exists $\lambda \in c a(\mathscr{B} a)^{+}$such that $\left\{\left|\bar{m}_{y^{\prime}}\right|: y^{\prime} \in B_{F^{\prime}}\right\}$ is $\lambda$-continuous (see [25, Theorem 4, pages 11-12]). Let $\left(f_{n}\right)$ be a sequence in $C_{b}(X, E)$ such that $\sup _{n}\left\|f_{n}\right\|=M<\infty$ and $f_{n}(t) \rightarrow 0$ for every $t \in X$. Let $\varepsilon>0$ be given. Then there exists $\delta>0$ such that $\sup \left\{\left|\bar{m}_{y^{\prime}}\right|(A): y^{\prime} \in\right.$ $\left.B_{F^{\prime}}\right\} \leq \varepsilon / 2 M$ whenever $\lambda(A) \leq \delta, A \in \mathscr{B} a$. Since $\tilde{f}_{n} \in B(\mathscr{B})$ for $n \in \mathbb{N}$, by the Egoroff theorem there exists $A_{\delta} \in \mathscr{B} a$ with $\lambda\left(X \backslash A_{\delta}\right) \leq \delta$ and $\sup _{t \in A_{\delta}} \tilde{f}_{n}(t) \rightarrow 0$. Choose $n_{\varepsilon} \in \mathbb{N}$ such that $\sup _{t \in A_{\delta}} \widetilde{f}_{n}(t) \leq \varepsilon / 2 \widetilde{m}(X)$ for $n \geq n_{\varepsilon}$.
Let $A \in \mathscr{B} a$. Note that $\bar{m}_{y^{\prime}}=\overline{m_{y^{\prime}}}$ for $y^{\prime} \in F^{\prime}$. Then by Lemma 11 and (ii), for $n \geq n_{\varepsilon}$ and $y^{\prime} \in B_{F^{\prime}}$ we get

$$
\begin{aligned}
& \left|y^{\prime}\left(\int_{A} f_{n} d \bar{m}\right)\right| \\
& \quad=\left|\int_{A} f_{n} d \bar{m}_{y^{\prime}}\right| \\
& \quad \leq \int_{A} \widetilde{f}_{n} d\left|\bar{m}_{y^{\prime}}\right| \leq \int_{X} \widetilde{f}_{n} d\left|\bar{m}_{y^{\prime}}\right| \\
& \quad=\int_{A_{\delta}} \tilde{f}_{n} d\left|\bar{m}_{y^{\prime}}\right|+\int_{X \backslash A_{\delta}} \widetilde{f}_{n} d\left|\bar{m}_{y^{\prime}}\right| \\
& \quad \leq \frac{\varepsilon}{2 \widetilde{m}(X)}\left|\bar{m}_{y^{\prime}}\right|\left(A_{\delta}\right)+M \cdot\left|\bar{m}_{y^{\prime}}\right|\left(X \backslash A_{\delta}\right) \\
& \quad \leq \frac{\varepsilon}{2 \widetilde{m}(X)}\left|m_{y^{\prime}}\right|(X)+M \cdot \frac{\varepsilon}{2 M} \leq \frac{\varepsilon}{2}+\frac{\varepsilon}{2}=\varepsilon .
\end{aligned}
$$

Hence $\left\|\int_{A} f_{n} d \bar{m}\right\|_{F} \leq \varepsilon$ for $n \geq n_{\varepsilon}$, as desired.

(iv) It follows from Theorem 14.

(v) It follows from (iii) and (iv).

Let $\mathscr{L}^{\infty}(\mathscr{B} a, E)$ stand for the Banach space of all bounded strongly $\mathscr{B} a$-measurable functions $g: X \rightarrow E$, equipped with the uniform norm $\|\cdot\|$. Assume that $m: \mathscr{B} \rightarrow \mathscr{L}(E, F)$ with $\widetilde{m}(X)<\infty$ is variationally semiregular. Then every $g \in$ $\mathscr{L}^{\infty}(\mathscr{B} a, E)$ is $m$-integrable (see [32, Definition 2, page 523 and Theorem 5, page 524]) and $\int_{X} g_{n} d m \rightarrow 0$ whenever $\left(g_{n}\right)$ is a uniformly bounded sequence in $\mathscr{L}^{\infty}(\mathscr{B} a, E)$ converging pointwise to 0 (see [33, Proposition 2.2]).

Recall that a series $\sum_{i=1}^{\infty} z_{i}$ in a Banach space $G$ is called weakly unconditionally Cauchy (wuc) if, for each $z^{\prime} \in G^{\prime}$, $\sum_{i=1}^{\infty}\left|z^{\prime}\left(z_{i}\right)\right|<\infty$. We say that a linear operator $T: G \rightarrow F$ is unconditionally converging if for every weakly unconditionally Cauchy series $\sum_{i=1}^{\infty} z_{i}$ in $G$, the series $\sum_{i=1}^{\infty} T\left(z_{i}\right)$ converges unconditionally in a Banach space $F$.

As an application of Theorem 17 we have the following result.

Corollary 18. Assume that $C_{b}(X) \otimes E$ is $\beta_{\sigma}$-dense in $C_{b}(X, E)$, where $E$ is a separable Banach space which contains no isomorphic copy of $c_{o}$. Let $T: C_{b}(X, E) \rightarrow F$ be a $\left(\beta_{\sigma},\|\cdot\|_{F}\right)$-continuous and strongly bounded operator. Then $T$ is unconditionally converging.

Proof. Assume that $\sum_{i=1}^{\infty} f_{i}$ is a wuc series in the Banach space $C_{b}(X, E)$. Hence $\sum_{i=1}^{\infty}\left|x^{\prime}\left(f_{i}(t)\right)\right|<\infty$ for each $t \in X$ and $x^{\prime} \in$ $E^{\prime}$ because $\delta_{t, x^{\prime}} \in C_{b}(X, E)^{\prime}$, where $\delta_{t, x^{\prime}}(f)=x^{\prime}(f(t))$ for $f \in C_{b}(X, E)$. It follows that $\sum_{i=1}^{\infty} f_{i}(t)$ is an unconditionally convergent series in $E$ for each $t \in X$ because $E$ contains no isomorphic copy of $c_{o}$ (see [34]). Let $g_{o}(t)=\lim _{n} S_{n}(t)$ for $t \epsilon$ $X$, where $S_{n}(t)=\sum_{i=1}^{n} f_{i}(t)$ for $t \in X, n \in \mathbb{N}$. Then $\sup _{n}\left\|S_{n}\right\|<$ $\infty$ because $\sum_{i=1}^{\infty} f_{i}$ is wuc (see [34]) and $S_{n} \in \mathscr{L}^{\infty}(\mathscr{B} a, E)$ because $E$ is assumed to be separable (see [2, Theorem 21, page 9]). Hence $g_{o} \in \mathscr{L}^{\infty}(\mathscr{B} a, E)$ (see [2, Theorem 10, page 6]).

Let $m \in M_{\sigma}\left(X, \mathscr{L}\left(E, F^{\prime \prime}\right)\right)$ be the representing measure of $T$ and let $\bar{m} \in M_{\sigma}(\mathscr{B} a, \mathscr{L}(E, F))$ be a unique extension of $m_{F} \in M_{\sigma}(\mathscr{B}, \mathscr{L}(E, F)$ ) (see Theorem 17). Since $\bar{m}$ is 
variationally semiregular, in view of [33, Proposition 2.2] we have

$$
\lim _{n} \sum_{i=1}^{n} T\left(f_{i}\right)=\lim _{n} \int_{X} S_{n} d \bar{m}=\int_{X} g_{o} d \bar{m} \in E .
$$

Hence $\sum_{i=1}^{\infty} T\left(f_{i}\right)=\int_{X} g_{o} d \bar{m}$. Finally, if $\left(n_{j}\right)$ is any permutation of $\mathbb{N}$, then $\lim _{n} \sum_{j=1}^{n} f_{n_{j}}(t)=g_{o}(t)$ for $t \in X$. Then $\sum_{j=1}^{\infty} T\left(f_{n_{j}}\right)=\int_{X} g_{o} d \bar{m}$, as desired.

Remark 19. A related result to Corollary 18 for strongly bounded operators on the space $C_{o}(X, E)$ of $E$-valued continuous functions vanishing at infinity defined on a locally compact space $X$ was obtained by Brooks and Lewis (see [21, Theorem 5.2]).

Recall that a Banach space $E$ is said to be a Schur space if every weakly convergent sequence in $E$ is norm convergent.

As a consequence of Theorem 17 we derive the following Dunford-Pettis type theorem for operators on $C_{b}(X, E)$.

Theorem 20. Assume that $C_{b}(X) \otimes E$ is $\beta_{\sigma}$-dense in $C_{b}(X, E)$, where $E$ is a Schur space. Let $T: C_{b}(X, E) \rightarrow F$ be a $\left(\beta_{\sigma},\|\cdot\|_{F}\right)$ continuous and strongly bounded operator. Then $T\left(f_{n}\right) \rightarrow 0$ in $F$ whenever $\left(f_{n}\right)$ is a $\sigma\left(C_{b}(X, E), M_{\sigma}\left(X, E^{\prime}\right)\right)$ convergent to 0 sequence in $C_{b}(X, E)$.

Proof. Assume that $f_{n} \rightarrow 0$ for $\sigma\left(C_{b}(X, E), M_{\sigma}\left(X, E^{\prime}\right)\right)$. Then according to [11, Corollary 5], we obtain that $\sup _{n}\left\|f_{n}\right\|<$ $\infty$ and $f_{n}(t) \rightarrow 0$ in $\sigma\left(E, E^{\prime}\right)$ for each $t \in X$. It follows that $\left\|f_{n}(t)\right\|_{E} \rightarrow 0$ for $t \in X$ because $E$ is supposed to be a Schur space. Using Theorem 17 we derive that $T\left(f_{n}\right) \rightarrow 0$ in $F$, as desired.

\section{Conflict of Interests}

The author declares that there is no conflict of interests regarding the publication of this paper.

\section{Acknowledgment}

The author wishes to thank the referee for useful remarks and suggestions that have improved the paper.

\section{References}

[1] N. Dinculeanu, Vector Measures, Pergamon Press, New York, NY, USA, 1967.

[2] N. Dinculeanu, Vector Integration and Stochastic Integration in Banach Spaces, John Wiley \& Sons, 2000.

[3] F. D. Sentilles, "Bounded continuous functions on a completely regular space," Transactions of the American Mathematical Society, vol. 168, pp. 311-336, 1972.

[4] R. F. Wheeler, "A survey of Baire measures and strict topologies," Expositiones Mathematicae, vol. 1, no. 2, pp. 97-190, 1983.

[5] D. Fontenot, "Strict topologies for vector-valued functions," Canadian Journal of Mathematics, vol. 26, no. 4, pp. 841-853, 1974.
[6] S. S. Khurana, "Topologies on spaces of vector-valued continuous functions," Transactions of the American Mathematical Society, vol. 241, pp. 195-211, 1978.

[7] S. S. Khurana and S. A. Choo, "Strict topology and P-spaces," Proceedings of the American Mathematical Society, vol. 61, no. 2, pp. 280-284, 1976.

[8] S. S. Khurana and S. I. Othman, "Convex compactness property in certain spaces of measures," Mathematische Annalen, vol. 279, no. 2, pp. 345-348, 1987.

[9] S. S. Khurana and S. I. Othman, "Completeness and sequential completeness in certain spaces of measures," Mathematica Slovaca, vol. 45, no. 2, pp. 163-170, 1995.

[10] S. S. Khurana and J. E. Vielma, "Strict topology and perfect measures," Czechoslovak Mathematical Journal, vol. 40, no. 1, pp. $1-7,1990$.

[11] S. S. Khurana and J. Vielma, "Weak sequential convergence and weak compactness in spaces of vector-valued continuous functions," Journal of Mathematical Analysis and Applications, vol. 195, no. 1, pp. 251-260, 1995.

[12] J. Aguayo-Garrido, "Strict topologies on spaces of continuous functions and $u$-additive measure spaces," Journal of Mathematical Analysis and Applications, vol. 220, no. 1, pp. 77-89, 1998.

[13] E. Granirer, "On Baire measures on D-topological spaces," Fundamenta Mathematicae, vol. 60, pp. 1-22, 1967.

[14] A. Katsaras and D. B. Liu, "Integral representations of weakly compact operators," Pacific Journal of Mathematics, vol. 56, no. 2, pp. 547-556, 1975.

[15] J. Aguayo-Garrido and M. Nova-Yanéz, "Weakly compact operators and $u$-additive measures," Annales Mathématiques Blaise Pascal, vol. 7, no. 2, pp. 1-11, 2000.

[16] J. Aguayo and J. Sanchez, "The Dunford-Pettis property on vector-valued continuous and bounded functions," Bulletin of the Australian Mathematical Society, vol. 48, no. 2, pp. 303-311, 1993.

[17] S. S. Khurana, "Integral representations for a class of operators," Journal of Mathematical Analysis and Applications, vol. 350, no. 1, pp. 290-293, 2009.

[18] I. Dobrakov, "On representation of linear operators on $C_{0}(T, X)$," Czechoslovak Mathematical Journal, vol. 21, no. 1, pp. 13-30, 1971.

[19] S. K. Mitter and S. K. Young, "Integration with respect to operator-valued measures with applications to quantum estimation theory," Annali di Matematica Pura ed Applicata, vol. 137, no. 4, pp. 1-39, 1984.

[20] P. Lewis, "Variational semi-regularity and norm convergence," Journal Für Die Reine Und Angewandte Mathematik, vol. 260, pp. 21-30, 1973.

[21] J. K. Brooks and P. W. Lewis, "Linear operators and vector measures," Transactions of the American Mathematical Society, vol. 192, pp. 139-162, 1974.

[22] J. K. Brooks and P. W. Lewis, "Operators on continuous function spaces and convergence in the spaces of operators," Advances in Mathematics, vol. 29, no. 2, pp. 157-177, 1978.

[23] A. Katsaras, "Continuous linear functionals on spaces of vectorvalued functions," Bulletin Société Mathématique de Grèce, vol. 15 , pp. 13-19, 1974.

[24] J. Hoffmann-Jörgënsen, "Vector measures," Mathematica Scandinavica, vol. 28, pp. 5-32, 1971.

[25] J. Diestel and J. J. Uhl, Vector Measures, vol. 15 of Mathematical Surveys, American Mathematical Society, Providence, RI, USA, 1977. 
[26] C. D. Aliprantis and O. Burkinshaw, Positive Operators, Academic Press, New York, NY, USA, 1985.

[27] A. Katsaras, "Spaces of vector measures," Transactions of the American Mathematical Society, vol. 206, pp. 313-328, 1975.

[28] G. Koumoullis, "Perfect, $u$-additive measures and strict topologies," Illinois Journal of Mathematics, vol. 26, no. 3, pp. 466-478, 1982.

[29] C. D. Aliprantis and O. Burkinshaw, Locally Solid Riesz Spaces with Applications to Economics, vol. 105 of Mathematical Surveys and Monographs, American Mathematical Society, Providence, RI, USA, 2003.

[30] S. S. Khurana, "Vector measures on topological spaces," Georgian Mathematical Journal, vol. 14, no. 4, pp. 687-698, 2007.

[31] P. W. Lewis, "Strongly bounded operators," Pacific Journal of Mathematics, vol. 53, no. 1, pp. 207-209, 1974.

[32] I. Dobrakov, "On integration in Banach spaces I," Czechoslovak Mathematical Journal, vol. 20, no. 95, pp. 511-536, 1970.

[33] M. Nowak, "Operators on the space of bounded strongly measurable functions," Journal of Mathematical Analysis and Applications, vol. 388, no. 1, pp. 393-403, 2012.

[34] C. Bessaga and A. Pełczyński, "On bases and unconditional convergence of series in Banach spaces," Studia Mathematica, vol. 17, pp. 151-164, 1958. 


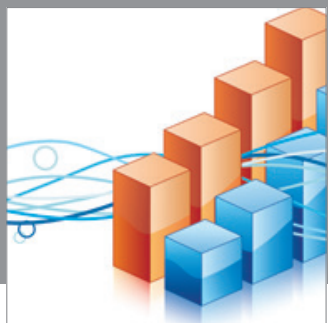

Advances in

Operations Research

mansans

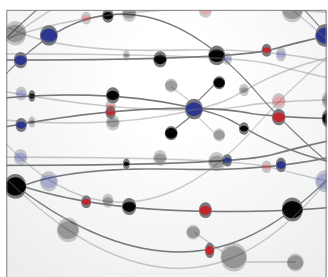

The Scientific World Journal
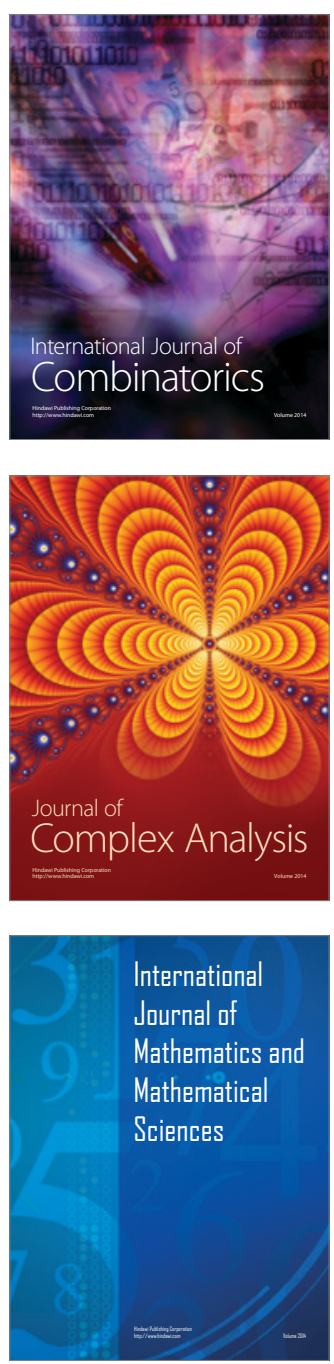
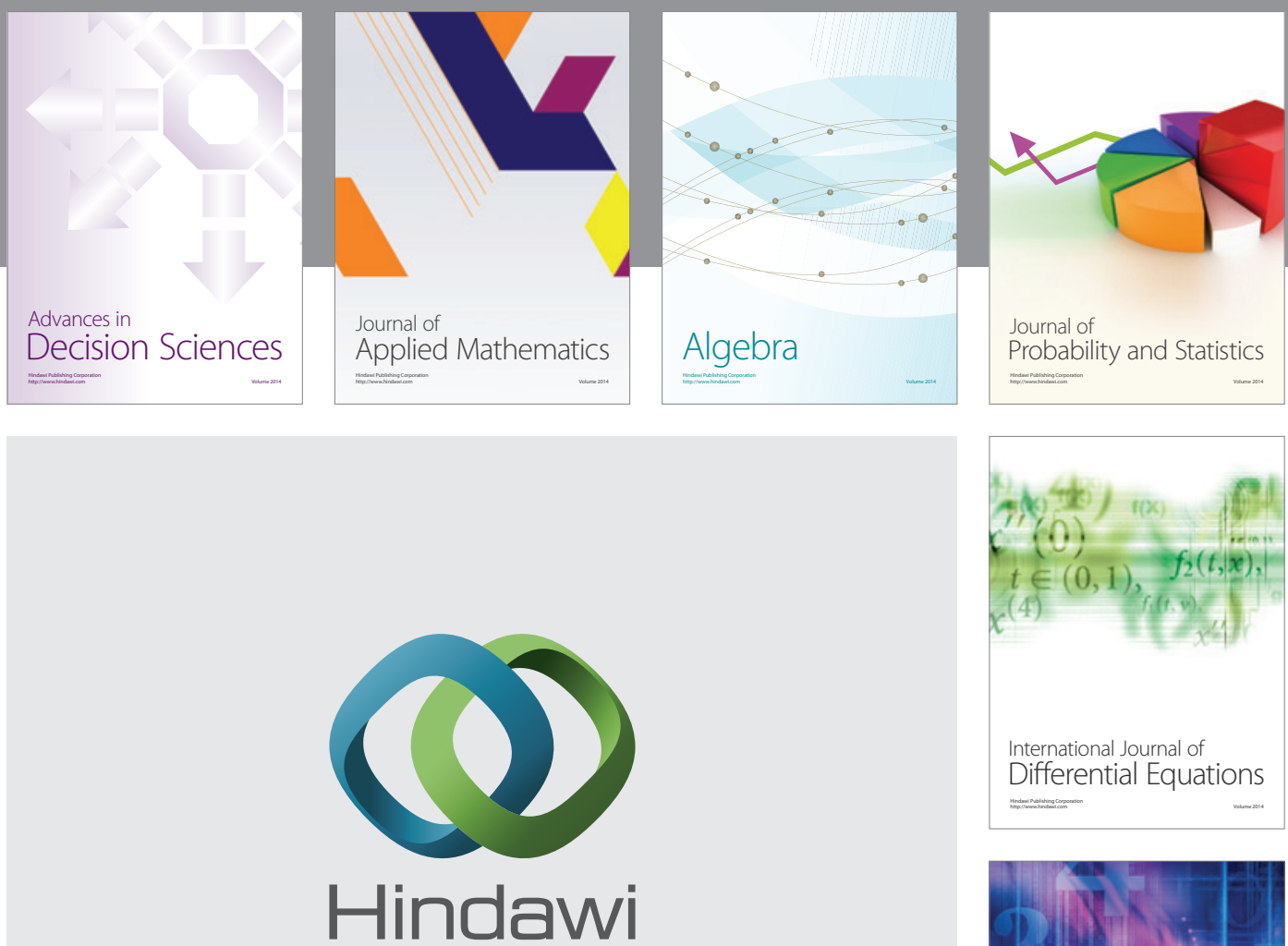

Submit your manuscripts at http://www.hindawi.com
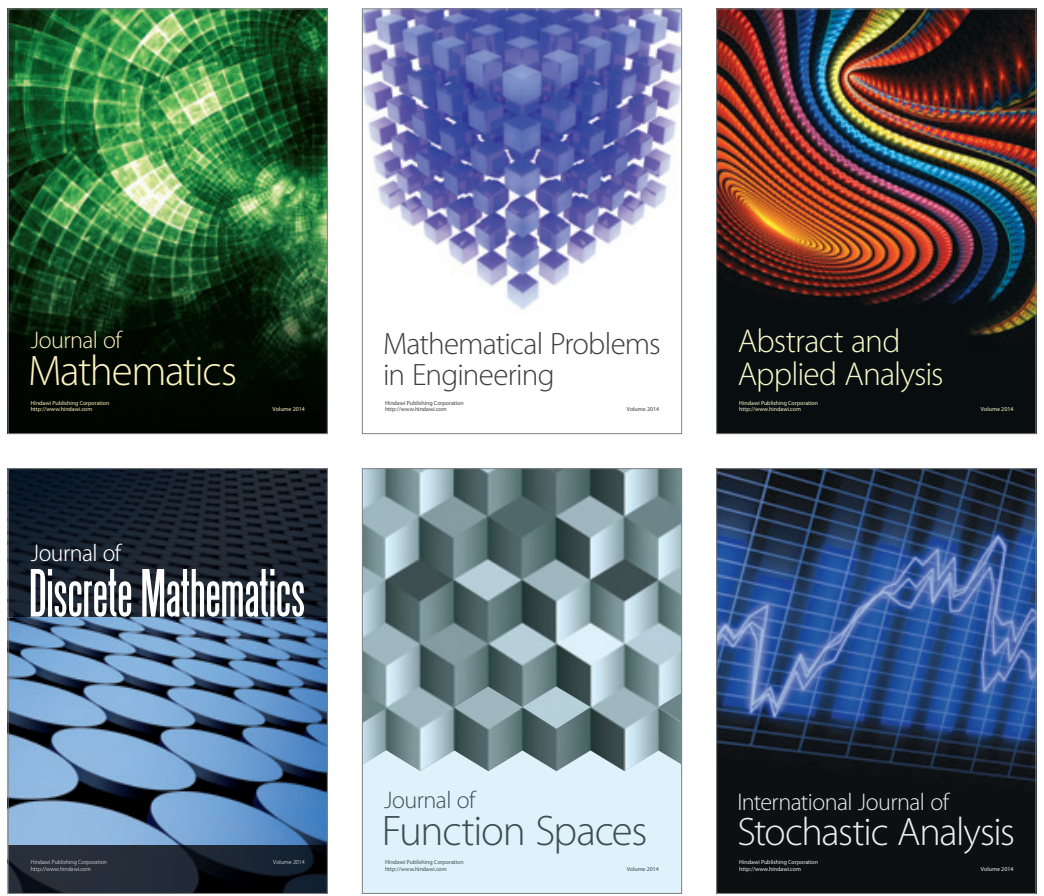

Journal of

Function Spaces

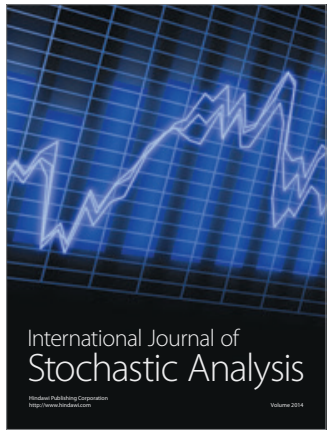

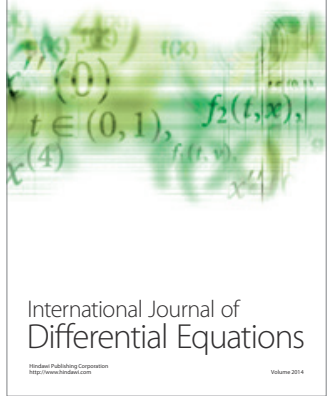
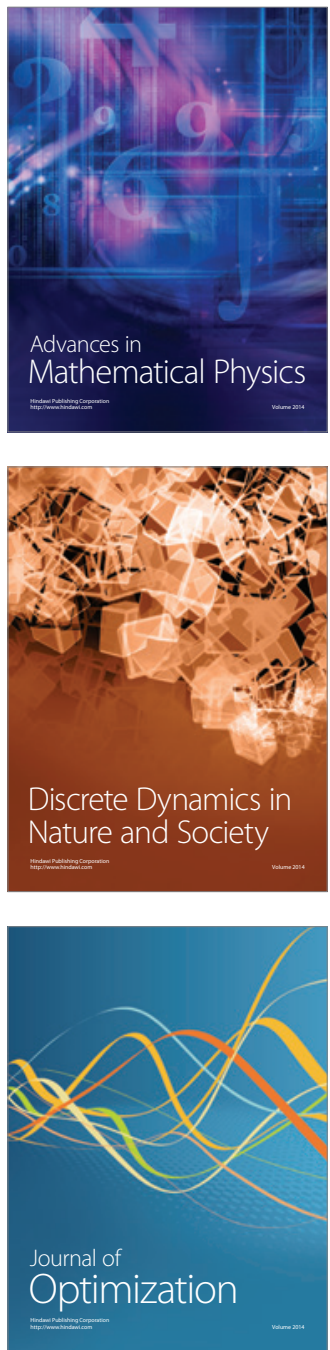